\title{
Clinical and Laboratory Approach to Diagnose COVID-19 Using Machine Learning
}

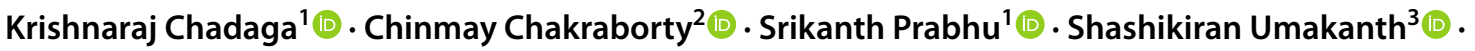 \\ Vivekananda Bhat ${ }^{1}\left[\right.$ ( Niranjana Sampathila ${ }^{4}$ (])
}

Received: 27 October 2021 / Revised: 17 December 2021 / Accepted: 23 December 2021 / Published online: 8 February 2022

(c) The Author(s) 2022

\begin{abstract}
Coronavirus 2 (SARS-CoV-2), often known by the name COVID-19, is a type of acute respiratory syndrome that has had a significant influence on both economy and health infrastructure worldwide. This novel virus is diagnosed utilising a conventional method known as the RT-PCR (Reverse Transcription Polymerase Chain Reaction) test. This approach, however, produces a lot of false-negative and erroneous outcomes. According to recent studies, COVID-19 can also be diagnosed using X-rays, CT scans, blood tests and cough sounds. In this article, we use blood tests and machine learning to predict the diagnosis of this deadly virus. We also present an extensive review of various existing machine-learning applications that diagnose COVID-19 from clinical and laboratory markers. Four different classifiers along with a technique called Synthetic Minority Oversampling Technique (SMOTE) were used for classification. Shapley Additive Explanations (SHAP) method was utilized to calculate the gravity of each feature and it was found that eosinophils, monocytes, leukocytes and platelets were the most critical blood parameters that distinguished COVID-19 infection for our dataset. These classifiers can be utilized in conjunction with RT-PCR tests to improve sensitivity and in emergency situations such as a pandemic outbreak that might happen due to new strains of the virus. The positive results indicate the prospective use of an automated framework that could help clinicians and medical personnel diagnose and screen patients.
\end{abstract}

\section{Graphical abstract}

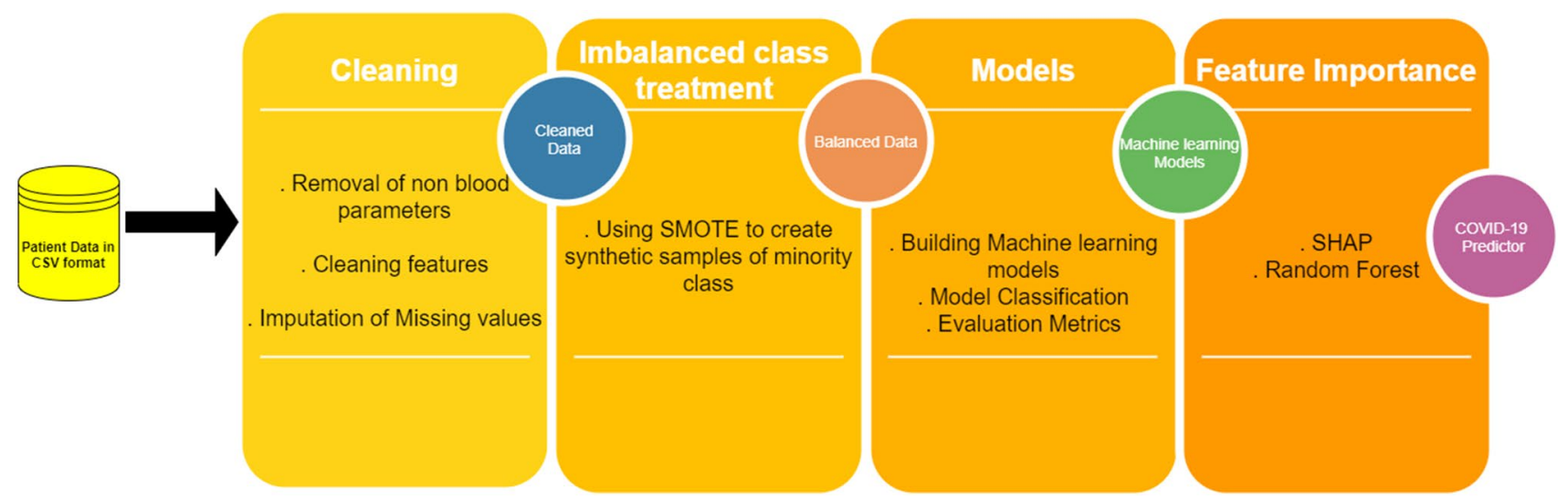

Keywords Artificial Intelligence $\cdot$ Machine Learning $\cdot$ COVID-19 $\cdot$ Blood tests $\cdot$ RT-PCR

Srikanth Prabhu

srikanth.prabhu@manipal.edu

Extended author information available on the last page of the article 


\section{Introduction}

The Coronavirus is an extremely dangerous infection transmitted by the Severe Acute Respiratory Coronavirus 2 (SARS-CoV-2) that has rapidly spread around the world. It has turned out to be an extremely fatal disease and is the reason for more than 500,000 deaths across 216 countries [1]. Every aspect of human activity has been impacted severely in all geographic territories and quick detection and treatment of this virus is extremely crucial to avert the escalation of this infectious virus. Currently, COVID-19 is commonly diagnosed using RT-PCR (Reverse Transcription Polymerase Chain Reaction) along with the Rapid Antigen tests (RAT) [2]. These tests are time-consuming and about $20 \%$ false negative rates have been observed [3]. A large number of underdeveloped nations do not have accessibility to RT-PCR testing kits. RAT testing is based on IgM/IgG antibodies. Low specificity (77.8\%) and sensitivity (18.8\%) have been the main drawbacks of this method [4]. Therefore, emphasis is being given to other methods of testing that might be more accessible and less expensive in the future. One of the most trending concepts in the modern world is Artificial Intelligence (AI). Various aspects such as Machine Learning (ML), modelling, statistics, simulations and algorithms are included in the above concept. It also contributes significantly to clinical and academic research [5]. Engineering, medical, psychology, sociology, hazard mitigation, multi-disciplinary science and other fields can efficiently make use of ML in the future. Numerous applications of Machine Learning (ML) have been utilized in activities such as sanitizing places with drones [6], tracking users using face recognition, drug development, automated robots delivering medicine and food, COVID-19 diagnosis, etc. According to the current literature, ML and hybridised models have been successfully applied in several domains of engineering [7-10], psychometric analysis [11, 12], medical and pharmaceutics [13-15], graph theory [16], and social sciences [17-19].

A considerable interest has been taken by various researchers in examining the field of AI and ML applications in battling this deadly virus by effectively deploying them in forecasting, diagnosis and prognosis, drug discovery and disease surveillance [20,21]. ML techniques have been deployed to help health care specialists with rapid, reliable and accurate detection of the novel coronavirus in this article. Computed Tomography Scans (CTScans) and chest X-ray images (XSR) images along with AI based medical imaging have been successfully used to detect the viral disease. Biomedical image analysis using AI has gained a lot of prominence and a lot of articles have been published with a sole focus on CT-Scans and X-rays
[22-25]. However, the radiation doses emitted during CTScans can cause cancer. High cost and availability of CTScanners is also an issue. Research has also taken place in exploring the use of cough sounds for COVID-19 diagnosis using NLP (Natural Language Processing) [26-28].

The blood and laboratory markers of COVID-19 patients can change drastically and these parameters can be used in the preliminary screening according to a numerous number of medical studies [29-33]. The presence of this infection can be confirmed by diagnosis, while a probabilistic indication of the disease's presence can be provided by a round of initial screening tests. It is very difficult for a doctor/physician to extract complete information from different laboratory blood tests. But, various patterns obtained from blood parameters can be easily differentiated by the AI models. Therefore, development of ML models that can diagnose COVID-19 has been explored by many ML researchers and enthusiasts [34-36].

The ML framework using blood tests for COVID-19 detection can lead to an accessible, less expensive, easy to use and faster alternative to time-consuming and expensive tests. Furthermore, these tests can be utilised in conjunction with RT-PCR testing to avoid false negatives. Blood test-based tests can be used in poor and underdeveloped countries that suffer from a lack of technology and laboratory supplies. This inexpensive system can also speed up testing and maintain a smooth flow of patients [37, 38]. The main findings and contributions of this article are given below:

- An exhaustive review of various ML applications that diagnose COVID-19 using various blood and laboratory markers.

- An in-depth data analysis that reveals crucial and critical blood markers that are key in diagnosing coronavirus.

- Different machine-learning models that accurately detect COVID-19 from a variety of clinical indicators.

- Shapley Additive Explanations (SHAP) and random forest technique were used to validate feature importance. It was observed that platelets, leukocytes, monocytes and eosinophils were the most critical markers that may signify the occurrence of coronavirus for our data.

- Additional information about the various blood parameters that are critical in the diagnosis of the novel COVID19 virus.

The aim and objective of this article is to introduce a ML based diagnosis framework that detects COVID-19 using routine blood parameters. Accuracy, recall, specificity, sensitivity, f1-score, AUC and brier score were the metrics used to evaluate our models to understand the advantages and disadvantages of the classifiers in this extensive study. 


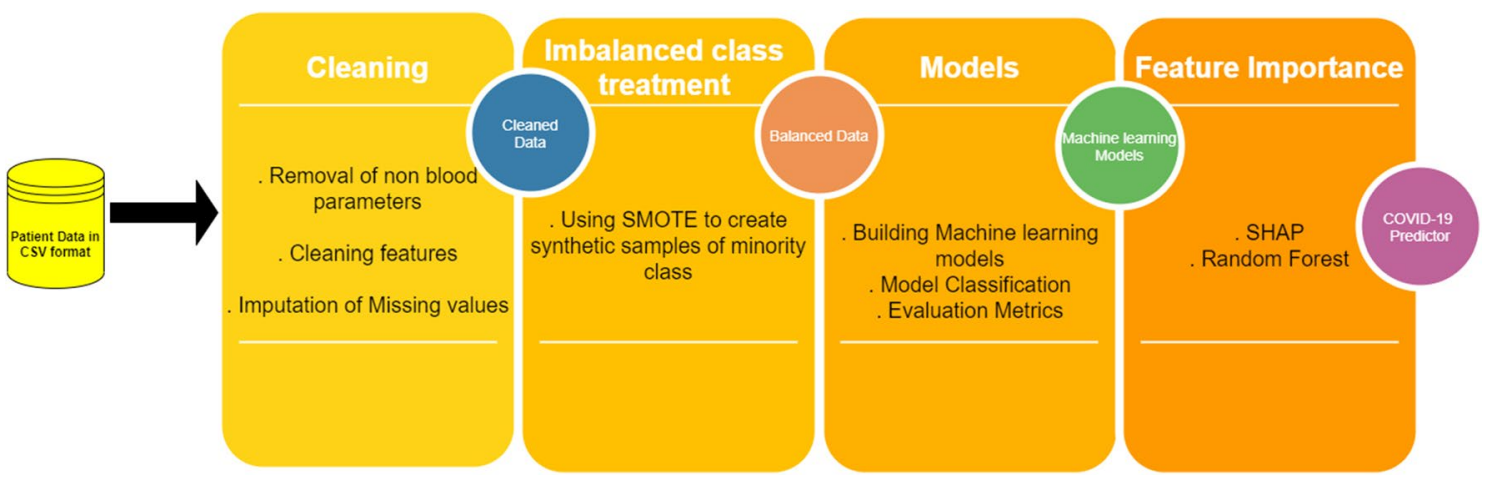

Fig. 1 Integral learning steps required for the development of ML classifiers

The Random forest classifier achieved the best results in diagnosing COVID-19 for the dataset that was available publicly from Hospital Israelita Albert Einstein, Brazil. The Synthetic Minority Oversampling Technique (SMOTE) was employed to prevent imbalance in the data distribution since the dataset was extremely unbalanced. The Shapley Additive Explanations (SHAP) and the random forest approaches were utilized to calculate importance of the features and Pearson's co-relation was used to find the various hidden co-relations between the various blood parameters and its relationship with this contagious virus. In this study, we first perform an extensive review of the existing literature in Sect. 2. Exploratory data analysis and the proposed design methodology are outlined in Sect. 3, followed by the evaluation of models in Sect. 4. Various challenges and the directions for future researches are described in Sect. 5. The article concludes in Sect. 6. Description of various techniques required for the design and development of the prospective ML models is given in Fig. 1.

\section{Related Work}

This section consolidates a number of ML researches that have been used to diagnose COVID-19. These infections are increasing at a rapid rate and it is of utmost importance to identify patients early to avoid the large scale spread of the contagious disease. RT-PCR is the existing standard procedure for diagnosing coronavirus and samples are accumulated from the respiratory tracts. Further, PCR amplification is conducted after the RNA has been extracted successfully using a predefined medical protocol. This unique method is still the golden standard for diagnosis. However, it still has a number of limitations. Specialist equipment and trained personnel are required to execute this test [39]. Testing a single sample is not feasible since it is very expensive and can take a lot of time ( 4 to $5 \mathrm{~h}$ ). To reduce costs, PCR machines are used with a number of samples. False negative rates have been found at a rate estimated to be between 3 and $30 \%$. [40]. These incorrect results are dangerous since the patient will not be isolated and can cause further spread of the disease. CT-Scans have been used as an alternative to PCR tests [41, 42]. However, they cannot confirm the exact diagnosis of this viral disease. CT-Scans are not available everywhere and they can also cause exposure to unnecessary radiation [43]. Hence, doctors do not recommend CT-Scans and chest radiographs (CXR) for every single patient [44]. Clinical and routine blood tests can be used as an inexpensive and quick means of COVID-19 detection. These accurate algorithms can be used efficiently, especially during a pandemic peak when there is an acute shortage of hospital resources [45, 46]. Validation of RT-PCR tests maybe be conducted to reduce false negatives and increase the sensitivity using these blood test classifiers [47, 48]. Some researchers have used one particular model, others have chosen multiple models and some of the predictive models are a combination of many models. Various ML models that diagnose COVID-19 are described below. The rest of the articles, along with their key characteristics, are described in Table 1.

Wu et al. [49] presented the first model that diagnosed COVID-19 from routine blood parameters. They used 11 parameters out of the initial 49 parameters for training the ML model. A combination of 235 (105 COVID-19) patients were used in this research. The accuracy, specificity and sensitivity obtained were $95.95 \%, 95.13 \%$, and $96 \%$ respectively for the external dataset. The blood parameters of 279 patients (177 COVID-19) from San Raffael Hospital were collected in the study [36]. Fourteen important blood parameters were given as input features to the various machinelearning classifiers. The accuracy obtained was $82-86 \%$ and the sensitivity obtained was $92-95 \%$. The paper also concluded that AST (Aspartate Aminotransferase), lymphocytes, LDH (Lactate dehydrogenase), WBC (White Blood Cells) and CRP (C-Reactive protein) were the most important diagnostic blood parameters. 
Table 1 List of ML models that diagnose COVID-19

\begin{tabular}{|c|c|c|c|c|c|c|c|c|}
\hline References & Source & Size & Total attributes & Models used & $\begin{array}{l}\text { Accuracy of } \\
\text { best model }\end{array}$ & $\begin{array}{l}\text { Sensitiv- } \\
\text { ity of best } \\
\text { model }\end{array}$ & $\begin{array}{l}\text { Specific- } \\
\text { ity of best } \\
\text { model }\end{array}$ & $\begin{array}{l}\text { AUC of best } \\
\text { model }\end{array}$ \\
\hline [57] & $\begin{array}{l}\text { Hospital Isra- } \\
\text { elita Albert } \\
\text { Einstein, } \\
\text { Brazil }\end{array}$ & $\begin{array}{l}\text { 5644, } 559 \\
\text { COVID-19 }\end{array}$ & 24 attributes & $\begin{array}{l}\text { MLP (Multi-layer } \\
\text { perceptron), } \\
\text { SVM, DT, NB }\end{array}$ & $95 \%$ & $96 \%$ & $93 \%$ & \\
\hline$[58]$ & $\begin{array}{l}\text { Three Open } \\
\text { access data- } \\
\text { sets }\end{array}$ & - & Many features & $\begin{array}{l}\text { Machine learning } \\
\text { and Deep Learn- } \\
\text { ing models }\end{array}$ & & $92 \%$ & $82 \%$ & $92 \%$ \\
\hline [59] & $\begin{array}{l}18 \text { hospitalls } \\
\text { from Zheji- } \\
\text { ang, China }\end{array}$ & 914 patients & 10 features & $\begin{array}{l}\text { LR, } \\
\text { SVM,DT,RF,RL }\end{array}$ & & $95 \%$ & $87 \%$ & $97 \%$ \\
\hline$[60]$ & $\begin{array}{l}\text { Tongji Hospi- } \\
\text { tal, China }\end{array}$ & 413 patients & $\begin{array}{l}\text { 21- categorical, } \\
21 \text { - continu- } \\
\text { ous }\end{array}$ & Xgboost & & $92.5 \%$ & $97.5 \%$ & \\
\hline$[61]$ & $\begin{array}{l}\text { West China } \\
\text { Hospital,m } \\
\text { China }\end{array}$ & 620 samples & 9 features & $\begin{array}{l}\text { Multi variate logis- } \\
\text { tic regression }\end{array}$ & - & - & - & - \\
\hline$[62]$ & $\begin{array}{l}11 \text { regions in } \\
\text { China }\end{array}$ & 659 patients & $\begin{array}{l}\text { Many bio- } \\
\text { chemical } \\
\text { and clinical } \\
\text { features }\end{array}$ & Decision trees & $89 \%$ & - & - & $88 \%$ \\
\hline [63] & $\begin{array}{l}\text { SMART hos- } \\
\text { pitals }\end{array}$ & - & - & NB, RF, SVM & $93.33 \%$ & - & - & - \\
\hline$[33]$ & $\begin{array}{l}\text { Hospital Isra- } \\
\text { elita Albert } \\
\text { Einstein Hos- } \\
\text { pital, Brazil }\end{array}$ & $\begin{array}{l}\text { 5644, } 559 \\
\text { COVID-19 } \\
\text { patients }\end{array}$ & $\begin{array}{l}\text { Many blood } \\
\text { parameters }\end{array}$ & $\begin{array}{l}\text { ERLX, an ensem- } \\
\text { ble learning } \\
\text { model }\end{array}$ & $99.60 \%$ & $98.72 \%$ & $98.99 \%$ & $99.38 \%$ \\
\hline$[64]$ & UK Biobank & 4510 patients & - & $\begin{array}{l}\text { Linear discrimi- } \\
\text { nant analysis }\end{array}$ & - & - & - & $97 \%$ \\
\hline$[65]$ & $\begin{array}{l}\text { Hospital Isra- } \\
\text { elita Albert } \\
\text { Einstein Hos- } \\
\text { pital, Brazil }\end{array}$ & $\begin{array}{l}5644 \text { patients } \\
598 \text { COVID-19 } \\
\text { patients }\end{array}$ & $\begin{array}{l}\text { Many blood } \\
\text { parameters }\end{array}$ & $\begin{array}{l}\text { RF, Shallow } \\
\text { learning, flexible } \\
\text { ANN }\end{array}$ & - & - & - & $95 \%$ \\
\hline$[66]$ & $\begin{array}{l}\text { Hospital Isra- } \\
\text { elita Albert } \\
\text { Einstein Hos- } \\
\text { pital, Brazil }\end{array}$ & $\begin{array}{l}5644 \text { patients } \\
598 \text { COVID-19 } \\
\text { patients }\end{array}$ & $\begin{array}{l}\text { Many blood } \\
\text { parameters }\end{array}$ & Er-CoV & - & $70 \%$ & $85 \%$ & $86 \%$ \\
\hline [67] & $\begin{array}{r}\text { Kepler Univer- } \\
\text { sity Hospital }\end{array}$ & 1357 patients & $\begin{array}{l}28 \text { unique } \\
\text { features }\end{array}$ & Random forest & $86 \%$ & - & - & $74 \%$ \\
\hline$[68]$ & $\begin{array}{l}\text { Three Brazilian } \\
\text { Hospitals }\end{array}$ & $\begin{array}{l}815(442 \\
\text { COVID-19) }\end{array}$ & 19 features & $\begin{array}{l}\text { ADA boost, Gradi- } \\
\text { ent boosting, } \\
\text { Random forest, } \\
\text { extreme gradient } \\
\text { boosting, SVM, } \\
\text { partial least } \\
\text { square }\end{array}$ & - & $96 \%$ & $93 \%$ & - \\
\hline [69] & - & 1521 patients & $\begin{array}{l}130 \text { clinical } \\
\text { features }\end{array}$ & $\begin{array}{l}\text { HUST-19 (CNN } \\
\text { based frame- } \\
\text { work) }\end{array}$ & $94 \%$ & - & - & - \\
\hline$[70]$ & $\begin{array}{l}\text { Oxford Univer- } \\
\text { sity hospitals }\end{array}$ & $\begin{array}{l}1,14,957- \\
\text { negative } \\
437-\text { COVID- } \\
19\end{array}$ & - & $\begin{array}{l}\text { Various ML clas- } \\
\text { sifiers }\end{array}$ & & $77 \%$ & $95 \%$ & $93 \%$ \\
\hline [71] & $\begin{array}{l}\text { Five hospitals } \\
\text { in New York }\end{array}$ & $\begin{array}{l}4098 \text { COVID- } \\
19 \text { patients }\end{array}$ & $\begin{array}{l}\text { Many blood } \\
\text { parameters }\end{array}$ & XGBoost & - & - & - & $89 \%$ \\
\hline [72] & - & 279 cases & 13 features & $\begin{array}{l}\text { KNN, DT, RF, } \\
\text { SVM, RF }\end{array}$ & - & - & - & $91 \%$ \\
\hline
\end{tabular}


Kukar et al. [50] utilized ML models to predict the presence of coronavirus using the laboratory and clinical markers of 160 COVID-19 patients hospitalised in the University Medical Centre Ljubljana in Slovenia. The sample size also included 5333 COVID-19 negative patients. The classifiers utilised were Random Forest (RF), DNN (Deep Neural Network), and XGBoost (Extreme Gradient Boosting), with XGBoost producing the best results. The average sensitivity and AUC (Area Under Curve) achieved by the various methods were $88.9 \%$ and $97 \%$ respectively. Hypoalbuminemia (low levels of albumin) was observed in patients.

Fernandes et al. [51] investigated the blood laboratory markers of 235 COVID-19 patients admitted in Israelita Albert Einstein Hospital, Brazil. Fifteen distinct blood parameters were utilised as features, and the Support Vector Machine (SVM) produced the optimal prediction. The AUC, sensitivity, and specificity obtained were $85 \%, 68 \%$, and $85 \%$, respectively. According to the study, the most critical blood indicators were lymphocytes, leukocytes, and eosinophils. Alves et al. [34] used three ML algorithms to diagnose coronavirus from routine blood parameters. The sample consisted of 84 COVID-19 patients along with 608 other patients. The Local Decision Tree Explainer (DTX), criteria graphs and the random forest were the models used for classification. The random forest algorithm achieved optimal predictions with an accuracy, f1-score, sensitivity, specificity and AUROC of $88 \%, 76 \%, 66 \%, 96 \%$ and $86 \%$ respectively.

Plante et al. [52] used ML models to rule out SARSCoV-2 using various clinical tests. 2183 PCR confirmed patients from 43 hospitals from the United States of America were included in this research. These models generate a risk score out of 10 ( 0 being minimal risk and 10 being maximum risk). The XGBoost model was the best performing model that achieved a sensitivity and an AUROC score of 95.9\% and 91\% respectively. Arpaci et al. [53] utilized 14 clinical characteristics to predict COVID-19 infection and the dataset included 114 confirmed COVID-19 cases from Taizhou hospital in China's Zhejiang province. Six distinct classifiers were employed and logistic regression produced the best results with $84.21 \%$ accuracy. Sobrinho et al. [54] used ML models to prioritize patients for testing based on various blood markers. The dataset consists of 55,676 patients along with 12 features. Eight different models were used for training/testing. Out of these, six models achieved high performance with the decision tree achieving the optimal results with an accuracy of $89.12 \%$. LDH and CRP were the most distinctive features that could diagnose COVID19 , according to [55]. 15 features were used for the seven deployed ML models that were combined together. They achieved an AUROC and sensitivity of $91 \%$ and $93 \%$ respectively. However, the specificity obtained was poor (64\%). In another study, Logistic regression (LR), neural network models and random forest (RF) were utilized in COVID-19 diagnosis [56]. Twenty-three clinical feature variables were utilised in the models described above. The dataset consisted of 536 (106 COVID-19) patients from Rennes-Academic Hospital, France. The LR model obtained the best results with an AUROC of $93 \%$.

\section{Materials and Methods}

\subsection{Dataset Description}

The case data for this research was procured from 5644 patients who were hospitalized in the Albert Einstein Israelita Hospital, Brazil. To totally anonymize the data, best practises and standards were employed. The clinical data had already been standardised to get the best normal distribution possible (standard deviation $=1$, mean $=0$ ). This dataset was made available publicly and is often updated for collaborative research [73]. It includes the blood test reports of all in-patients who have been tested for Sars-CoV-2 virus (both positive and negative). 111 features that include various urine, blood and other medical tests of 5644 patients are included in this publicly available data. However, the dataset is extremely unbalanced, with very few positive cases (558) compared to a large number of negative cases. The various blood parameters include haemoglobin, haematocrit, platelets, red blood cells, leukocytes, lymphocytes, basophils and many more. Urine tests and tests for other contagious diseases were also included. RT-PCR results were used to confirm the patients' diagnosis and were represented as dichotomous ground truth values (positive/negative).

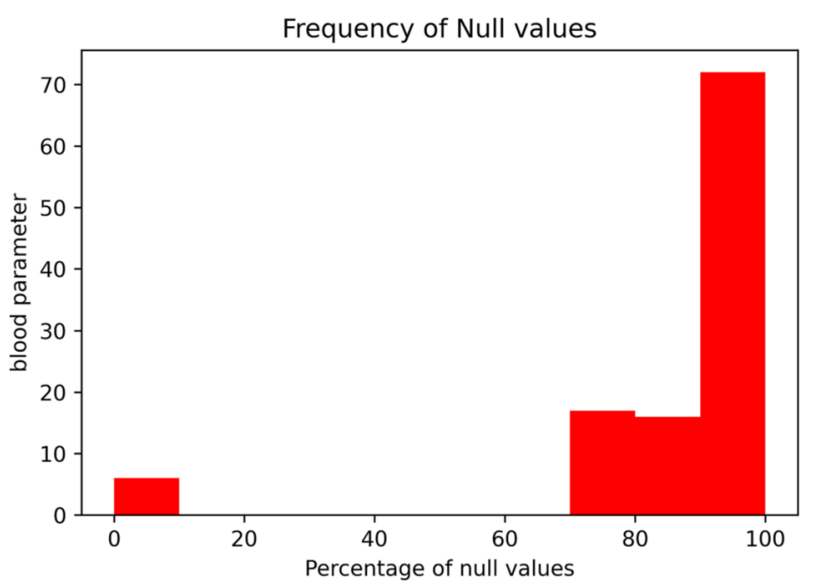

Fig. 2 Null values present in attributes 
Table 2 Feature description of the final selected parameters

\begin{tabular}{|c|c|c|c|c|}
\hline Sl.no & Abbreviation & Feature & Description & References \\
\hline 1 & AGE & Patient age quantile & Specifies the age of the individual & - \\
\hline 2 & MPV & Mean platelet volume & $\begin{array}{l}\text { Mean size of platelets presents in blood. It is known to increase in the } \\
\text { presence of COVID-19 }\end{array}$ & {$[74]$} \\
\hline 3 & $\mathrm{RBC}$ & Red blood cells & $\begin{array}{l}\text { The bone marrow produces fresh red blood cells. The red blood cell car- } \\
\text { ries oxygen and removes carbon dioxide from the body }\end{array}$ & {$[75]$} \\
\hline 4 & LYM & Lymphocytes & $\begin{array}{l}\text { These are part of the person's immune system and are created by the lymph } \\
\text { nodes and bone marrow. They tend to decrease for severe COVID-19 } \\
\text { patients }\end{array}$ & [76] \\
\hline 5 & $\mathrm{MCHC}$ & $\begin{array}{l}\text { Mean Corpuscular haemoglobin } \\
\text { concentration }\end{array}$ & Average quantity of haemoglobin present in each of the red blood cells & [77] \\
\hline 6 & WBC & Leukocytes & $\begin{array}{l}\text { They are also called white blood cells. They defend the body against } \\
\text { various infections and threats. The count has increased in COVID-19 } \\
\text { patients according to numerous studies }\end{array}$ & [78] \\
\hline 7 & BAY & Basophils & They are a part of white blood cells & [79] \\
\hline 8 & EOS & Eosinophils & $\begin{array}{l}\text { They help in promoting inflammation that controls the infection. Eosino- } \\
\text { phil count is reduced for COVID-19 patients }\end{array}$ & [79] \\
\hline 9 & $\mathrm{MCV}$ & Mean Corpuscular volume & $\begin{array}{l}\text { Average volume of red blood cells. They increase or decrease depending } \\
\text { on the average red cell size }\end{array}$ & [74] \\
\hline 10 & $\mathrm{MON}$ & Monocytes & They are white blood cells that focus on healing and repair & {$[80]$} \\
\hline 11 & PLT & Platelets & $\begin{array}{l}\text { They form clots and prevent bleeding. COVID-19 patients often have mild } \\
\text { thrombocytopenia }\end{array}$ & {$[81]$} \\
\hline 12 & RBCDW & Red blood cell distribution width & The range of volume and size of red blood cells & [75] \\
\hline 13 & - & Has_disease & $\begin{array}{l}\text { A variable that has been created by combining all the other disease col- } \\
\text { umns for this research. It specifies whether the patient suffers from other } \\
\text { viral diseases }\end{array}$ & - \\
\hline
\end{tabular}

\subsection{Data Pre-processing and Co-relation Analysis}

Missing values imputation, elimination of outliers and balancing the data are the three major phases in data preparation. Our dataset contained a lot of null values and Fig. 2 shows that over $90 \%$ of the blood parameters had a lot of missing values.

Imputing those values with statistical parameters (mean, median, mode) would render the model useless. Therefore, all the columns that contained at least $90 \%$ of the null values were removed. After the removal, there were 39 attributes left. The parameter "parainfluenza 2 " had only one value (variance $=0$ ) and was dropped. 3596 rows of 5644 cases had null values above $80 \%$. The dataset was trimmed further and all patient records were deleted that had more than 26 null values and all attributes and tests not related to COVID-19 were removed. The presence of antigens was mentioned in at least nineteen columns and the values were binary. These columns were used to confirm whether the patient tested positive for other viral infections such as Adenovirus, Parainfluenza, Metapneumovirus, etc. The results of these tests were combined together to form an attribute called "has-disease" and this attribute suggested if at least one of the respiratory infections were present. The dataset was already normalized except for a single column named "patient age quantile" and the values ranged from 1 to 19. The magnitude of the features can affect the results drastically in some AI algorithms. Hence, the age parameter was normalized in the range $[-3,3]$ to prevent the impact of attributes with various scales.

After data pre-processing, 18 columns and 602 rows remained. The final set of features that were chosen are described in Table 2. The dataset contained 84 positive and 518 negative cases confirmed by RT-PCR tests. Thus, the dataset still had the problem of severe data imbalance (1:6 ratio). The proposed model uses the SMOTE technique that is available in the "imblearn" python library. This innovative technique balances the dataset by oversampling the minority-class instances.

After completing the process of feature engineering, we proceed to feature selection. Pearson's co-relation coefficient (PCC) was used to evaluate the co-relation between the attributes to remove the non-essential and redundant blood markers as shown in Fig. 3. Features that showed strong co-relations that indicated COVID-19 were also observed. It was seen that eosinophils, platelets, leukocytes and the has_disease attribute showed a negative corelation (The values of these blood parameters decreased for COVID-19 patients), while monocytes, haemoglobin, red blood cells and age showed a slight positive co-relation 


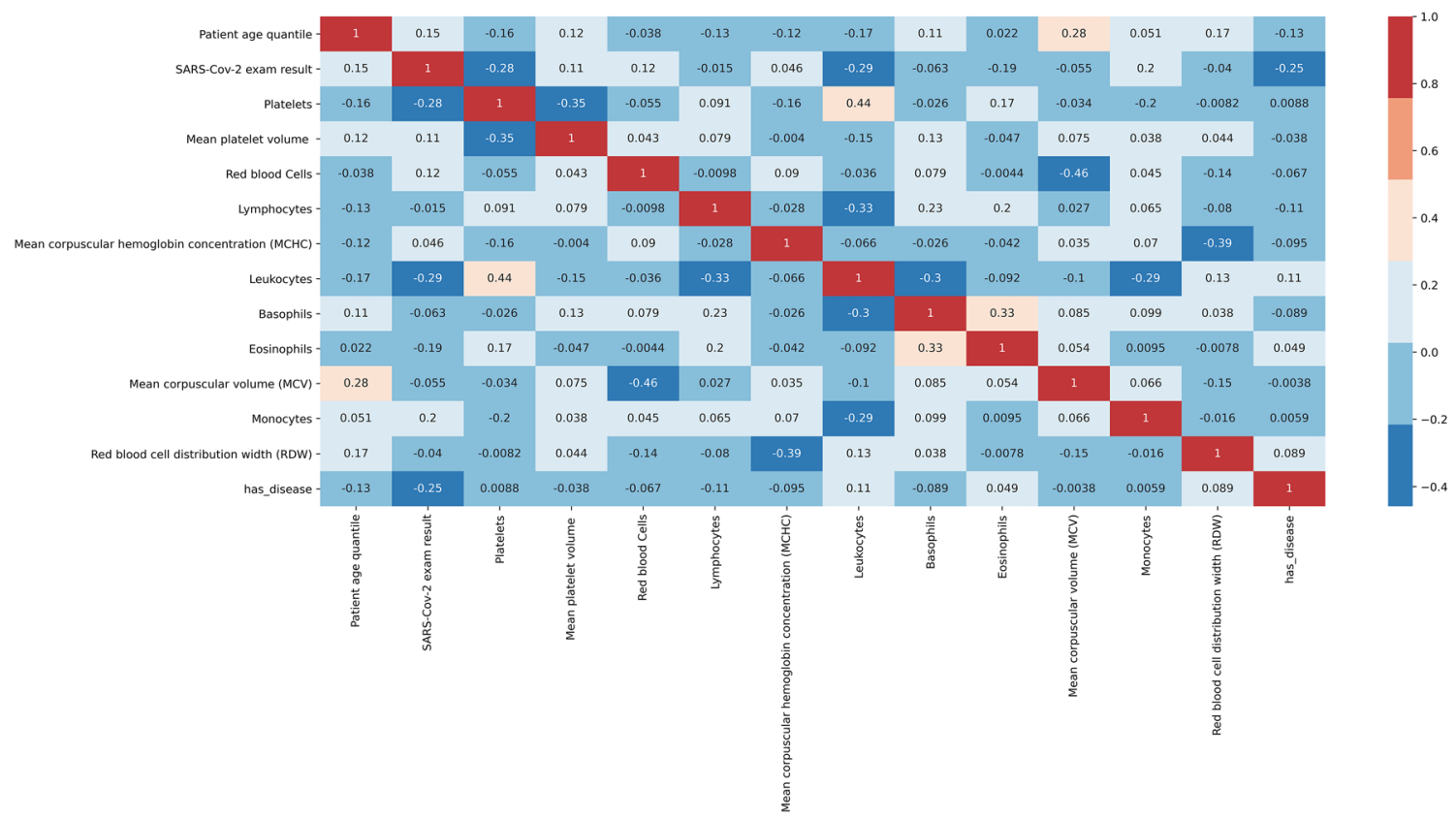

Fig. 3 Pearson co-relation matrix

Table 3 Correlation coefficient and $r$ value of the final blood parameters

\begin{tabular}{llcl}
\hline Dependent features & Result Label & $r$ value & Relationship co-relation \\
\hline Age & RT-PCR test & 0.15 & Weak positive correlation \\
MPV & RT-PCR test & 0.11 & Weak positive correlation \\
RBC & RT-PCR test & 0.12 & Weak positive correlation \\
LYM & RT-PCR test & -0.015 & Very weak negative correlation \\
MCHC & RT-PCR test & 0.046 & Very weak positive correlation \\
WBC & RT-PCR test & -0.29 & Weak negative co-relation \\
BAY & RT-PCR test & -0.063 & Very weak negative correlation \\
EOS & RT-PCR test & -0.19 & Weak negative co-relation \\
MCV & RT-PCR test & -0.055 & Very weak negative correlation \\
MON & RT-PCR test & 0.2 & Weak positive correlation \\
PLT & RT-PCR test & -0.28 & Weak negative co-relation \\
RBCDW & RT-PCR test & -0.04 & Very weak negative correlation \\
Has_Disease & RT-PCR test & -0.25 & Weak negative co-relation \\
\hline
\end{tabular}

(The values of these blood parameters increased for COVID-19 patients) as described in Table 3. Some characteristic parameters had a very high degree of interdependence. To reduce noise, we must remove parameters with a high degree of co-linearity between them. Haematocrit and haemoglobin had a co-relation factor of 0.97 between them and also had a strong positive relationship with red blood cells $(0.87$ and 0.84$)$. We decided to retain red blood cells since they had the highest co-relationship with the target variable. MCV (Mean Corpuscular volume) and $\mathrm{MCH}$ (Mean Corpuscular Height) were the other two highly associated variables. MCV was retained since it was more co-related to the target label $(-0.055 \mathrm{vs}-0.028)$.

\subsection{Methodology}

Since they are highly efficient with imbalanced data, xgboost, random forest and logistic regression were used as cutting-edge prediction models in this research. The KNN algorithm was also tested.

Random forest (RF) is a decision tree agglomeration approach that constructs many trees using a resampling procedure known as bagging (bootstrap aggregation) [82] Resampling with replacement is used to create a huge number of decision trees. Every tree's node is divided using a subset of the tree's characteristics that are chosen at random. A simple unweighted majority vote is used to 
determine the most often predicted class for new data from the (aggregated) decision trees. When more trees are introduced, random forests do not overfit. Instead they provide a limiting value of the generalisation error, as indicated in Eq. 1. The number of trees used for classification was varied with the following values $(10,50,100,200,500)$, split ratio of $(1,2,4,8,16,24)$ and minimum leaf nodes of $(1,2,5,10,15,30)$.

$$
P x, y\left(P g(h(X, 0)=Y) \max _{j=Y} P g(h(X, 0)=j)<0\right)
$$

By trying to compare an unlabelled data point to the training dataset, the K-nearest-neighbour (KNN) classifier improves considerably. It finds the $\mathrm{K}$ most related data-points, which are termed as KNNs [83]. A metric that measures distance such as Euclidean or Manhattan distance is widely utilized to determine proximity. This technique then assigns the given data point to the KNN's most familiar class. The number of potential nearest neighbours for $\mathrm{KNN}$ chosen were $(2,3,5,8,10,12,15,20)$. XGBoost is an ensemble approach to build a series of trees successively [84]. A tree's performance is enhanced in each iteration based on the preceding iteration's results. The three components involved in any boosting algorithm are a loss function, an additive model and a weak learner (e.g., a decision tree). XGBoost used the same parameters as random forests with an additional learning rate parameter that was varied with the following values $(0.01,0.05,0.1)$. LR algorithm estimates the maximum probability of datapoints pertaining to a particular label based on the values of the laboratory markers that are independent in nature $[85,86]$. The model can then be used to make predictions that a data-point belongs to a particular label. The sigmoid function is commonly utilized to generate a logistic regression model. The data points are expected to follow a linear function. The following is a description of LR. $\log (P(X) / 1-P(X))=\beta_{0}+\beta_{1} X$

where $P$ is the maximum probability that $X$ is a member to class $C$ and $\beta_{0}$ and $\beta_{1}$ are the parameters of the model. Testing was done using a ridge regression penalty of $(11,12)$ and a sparsity of $(100,10,1,0.1,0.01,0,001)$.

The SMOTE [87] algorithm was then utilized to train every classifier. This approach synthetically oversamples minority-class data, producing the same occurrences in the training data for the positive and negative classes. As demonstrated in Fig. 4, this strategy resamples by producing an optimal synthetic sample from the $\mathrm{k}$ neighbours adjacent to the model. For this research, we used a set of $k=3$ neighbours. We then selected the optimal of the five models produced for each classifier and retrained them in five separate iterations using their hyperparameters to assess their generalizability. We divided the dataset into 80 per cent for model training and 20 percent for model testing. With the imbalanced data in mind, we reran the SMOTE algorithm, but this time only for the training data, synthetically super sampling the minority-class data for each of the iterations. The overall proposed methodology is pictorially shown in Fig. 5.

\section{Results and Discussions}

This section assesses the proposed machine-learning models and examines the outcomes. The first subsection discusses about the significance of various metrics used in our research, the second section compares the performances of various models using the above metrics. Feature importance using SHAP and random forest are examined in subsection three. Discussions on the important blood parameters that can diagnose COVID-19 are portrayed in the last subsection.
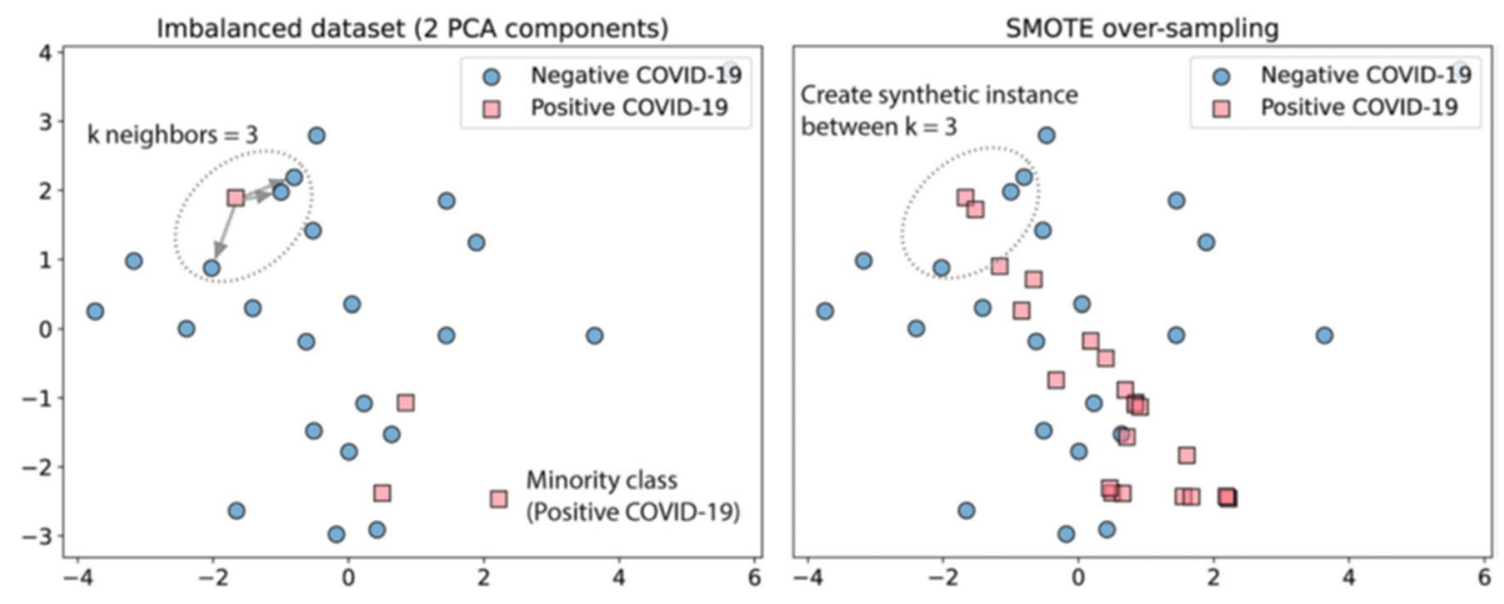

Fig. 4 An example of synthetic sampling by SMOTE overall flow diagram is given below [34] 
Fig. 5 Block diagram describing the proposed method for the classification of COVID-19 based on blood sample data

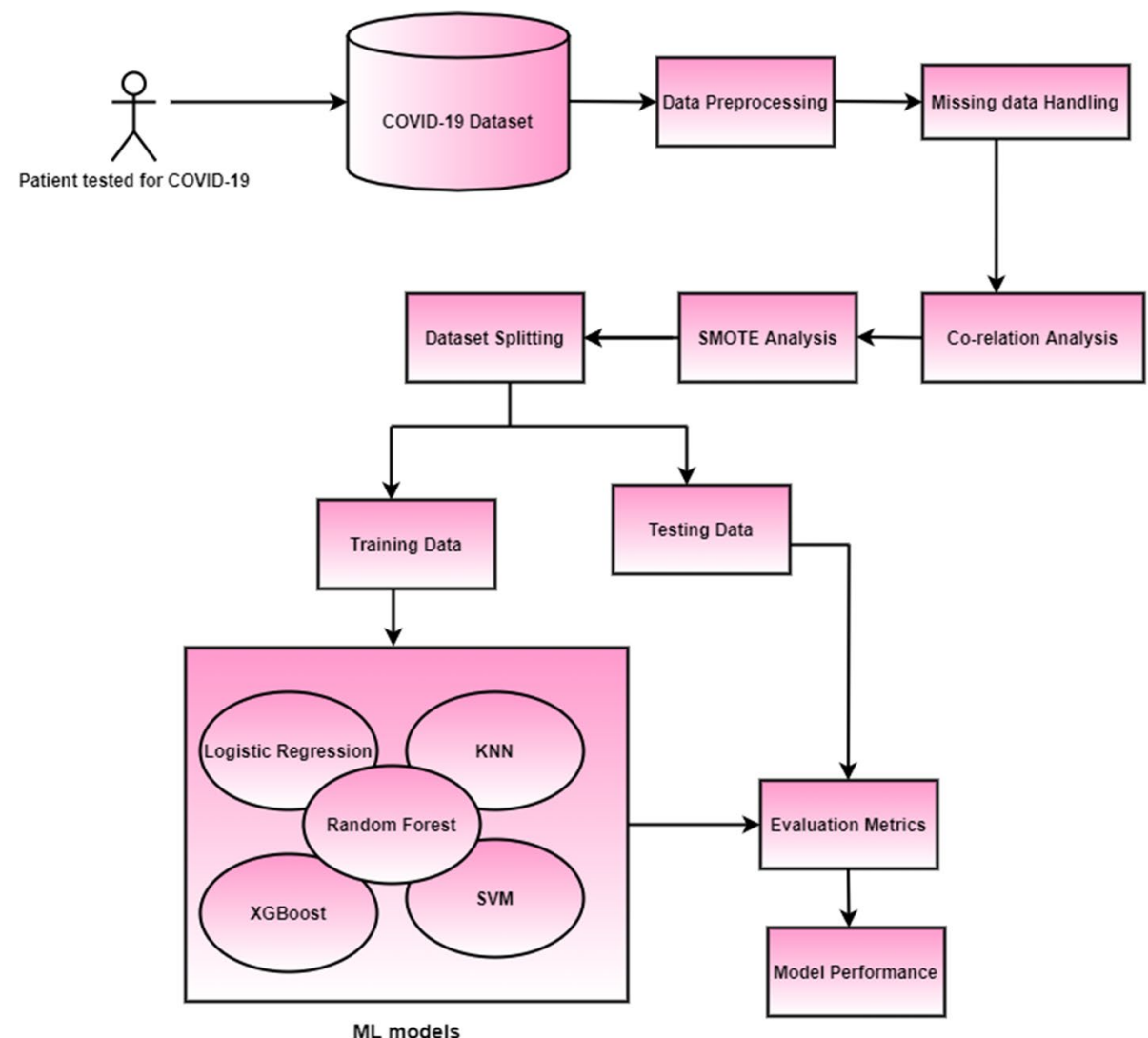

Table 4 Classification results

\begin{tabular}{|c|c|c|c|c|c|c|c|}
\hline Model & Accuracy & Specificity & Sensitivity & F1-score & AUC & Brier score & Best parameters \\
\hline Simple random forest & 0.60 & 0.71 & 0.33 & 0.66 & 0.69 & 0.23 & - \\
\hline Random forest after feature selection & 0.89 & 0.97 & 0.35 & 0.84 & 0.90 & 0.115 & - \\
\hline $\begin{array}{l}\text { Random forest after hyper parameter } \\
\text { tuning (Randomized searchCV) }\end{array}$ & 0.88 & 0.96 & 0.41 & 0.84 & 0.92 & 0.115 & $\begin{array}{l}\text { \{'n_estimators': } 10 \text {, 'min_samples_ } \\
\text { split': } 2 \text {, 'min_samples_leaf': } 2, \\
\text { 'max_features': } 10 \text {, 'max_depth': } \\
128\}\end{array}$ \\
\hline $\begin{array}{l}\text { Optimal random forest (After } \\
\text { SMOTE) }\end{array}$ & 0.92 & 0.96 & 0.71 & 0.85 & 0.92 & 0.082 & $\begin{array}{l}\text { \{'n_estimators': } 500, \text { 'min_samples_ } \\
\text { split': 4, 'min_samples_leaf': } 1 \text {, } \\
\text { 'max_features': '8', 'max_depth': } \\
32\}\end{array}$ \\
\hline Optimal LR & 0.85 & 0.87 & 0.70 & 0.81 & 0.89 & 0.157 & \{'penalty': '12', 'C': 100\} \\
\hline Optimal KNN & 0.75 & 0.77 & 0.59 & 0.73 & 0.68 & 0.25 & $\begin{array}{l}\text { \{'weights': 'distance', 'p': } 1, \text { 'n } \\
\text { neighbors': } 2 \text { \} }\end{array}$ \\
\hline Optimal XGBoost & 0.88 & 0.93 & 0.65 & 0.83 & 0.88 & 0.123 & $\begin{array}{l}\text { \{'n_estimators': } 100, \text { 'max_depth': } \\
\text { 8, 'gamma': } 0, \text { 'colsample_bytree': } \\
0.8\}\end{array}$ \\
\hline
\end{tabular}

\subsection{Performance Metrics}

Our prediction models are evaluated using a variety of performance indicators. The reliability of these models was assessed using the following performance indicators: AUC, accuracy, sensitivity, specificity, f1-score, and brier score. The confusion matrix is used to determine true positives' (TP), false positives' (FP), false negatives' (FN) and true negatives' (TN) as indicated in Table 4. The cases of TP occur when COVID19 patients are correctly predicted and the cases of TN occur when COVID-19 negative patients are correctly predicted. The number of cases that are predicted incorrectly are determined by false positives and false negatives. 
Accuracy The fraction of accurately predicted cases (Both COVID-19 negative and positive) in the entire data. The accuracy of a model in percentage is calculated using the following formula:

Accuracy $=\frac{\mathrm{TP}+\mathrm{TN}}{\mathrm{TP}+\mathrm{TN}+\mathrm{FP}+\mathrm{FN}}$

Specificity It defines the percentage of genuine negatives correctly predicted by a model. In our research, the total proportion of patients who were not infected with COVID-19 and were correctly predicted as negative cases by the classifier. A classification model with good specificity has a high TN and less FP rates. The formula for calculating specificity is presented in Eq. (2):

Specificity $=\frac{\mathrm{TN}}{\mathrm{TN}+\mathrm{FP}}$

Sensitivity (Recall) It is the total percentage of true positives for the dataset. In our research, the percentage of actual coronavirus patients who were accurately identified as COVID19 patients by the classifiers. A model with good sensitivity always has a high number of TP and less FN values. Equation (3) is used to calculate sensitivity.

Sensitivity $=\frac{\mathrm{TP}}{\mathrm{TP}+\mathrm{FN}}$

F1-Score It is a metric that measures model performance based on precision and sensitivity. It's used to assess binary classification algorithms that categorise examples as either "positive" or "negative." The F1-score is also the harmonic mean of the model's recall and precision. It is calculated using the formula:

$F 1-$ score $=2 \times \frac{(\text { Precision } \times \text { Recall })}{\text { Precision }+ \text { Recall }}$

ROC Curve The receiver operating characteristic (ROC) curve portrays the relationship between true positive rate (TPR) and the false positive rates (FPR). The area under curve (AUC) represents the area within the ROC curve and indicates how well the classifier distinguishes between its two categories. The higher the AUC, the better the predictions of the models.

Brier Score Brier score is a metric for assessing the quality of a probability score that has been forecasted. This is identical to the mean squared error. However, it only applies to prediction probability scores with values ranging from 0 to 1 . A perfect accurate sample will have a brier score of 0 and 1 represents perfect inaccuracy. It is calculated using the formula:

$\mathrm{BS}=\frac{1}{N} \sum_{t=1}^{N}\left(f_{t}-O_{t}\right)^{2}$

where $N=$ number of samples, $f_{t}=$ forecast probability, $O_{t}=$ is the actual outcome.

Table 5 Normalized confusion matrices for test data set

\begin{tabular}{|c|c|c|c|}
\hline \multirow[t]{2}{*}{ (a) Random forest } & & \multicolumn{2}{|l|}{ Actual } \\
\hline & & Negative & Positive \\
\hline \multirow[t]{2}{*}{ Predicted } & Negative & 0.94 & 0.06 \\
\hline & Positive & 0.41 & 0.59 \\
\hline \multirow[t]{2}{*}{ (b) Logistic regression } & & \multicolumn{2}{|l|}{ Actual } \\
\hline & & Negative & Positive \\
\hline \multirow[t]{2}{*}{ Predicted } & Negative & 0.87 & 0.13 \\
\hline & Positive & 0.30 & 0.70 \\
\hline \multirow[t]{2}{*}{ (c) $\mathrm{KNN}$} & & \multicolumn{2}{|l|}{ Actual } \\
\hline & & Negative & Positive \\
\hline \multirow[t]{2}{*}{ Predicted } & Negative & 0.70 & 0.30 \\
\hline & Positive & 0.41 & 0.59 \\
\hline \multirow[t]{2}{*}{ (d) XGBoost } & & \multicolumn{2}{|l|}{ Actual } \\
\hline & & Negative & Positive \\
\hline \multirow[t]{2}{*}{ Predicted } & Negative & 0.93 & 0.07 \\
\hline & Positive & 0.47 & 0.53 \\
\hline
\end{tabular}

For each actual class, the corresponding row sum is 1.0 


\subsection{Evaluation of Predictive Models}

Early COVID-19 prediction can help decrease the significant load on health care facilities by assisting in the diagnosis of infected patients. In this research, RF, LR, KNN and XGBoost supervised classifiers were utilized for the prediction of the deadly virus. The performances of the above models are depicted in Table 5. Randomized search technique was utilized to obtain the optimal parameters for the evaluation of the classifiers. The SMOTE technique was then applied on each of the above five models. COVID-19 positive patients(minority-class) were synthetically oversampled using this strategy. This resulted in more balance in the training data between the positive and negative classes. A total of $k=3$ neighbours were chosen for this specific task. We built final models using the fivefold cross validation, which specified the number of external splits. As a result, we selected the perfect four models and retrained them in five iterations using the given hyperparameters to assess their generalizability. We split the data in half for each cycle, using $80 \%$ for training and the rest as testing.

In comparison to other models, the random forest model produced good results. After data pre-processing and SMOTE analysis, the best model had a 92\% accuracy. The accuracy of KNN, logistic regression and XGBoost were $75 \%, 85 \%$ and $88 \%$ respectively. The percentage of COVID19 positive patients properly predicted is revealed by sensitivity (recall). Our models, however, did not do well in terms of sensitivity. A maximum sensitivity of $71 \%$ was achieved using the random forest technique. Although the sensitivity attained was not particularly impressive, it was nevertheless adequate given the dataset's complexity. The number of COVID-19 negative patients correctly detected is calculated using specificity. Random forest obtained the highest specificity of 96\%. KNN, LR and XGBoost obtained a specificity of $77 \%, 87 \%$ and $93 \%$ respectively. Specificity define the number of coronavirus negative patients identified correctly. A maximum specificity of $96 \%$ was obtained by the RF classifier. The specificity of the LR, KNN and XGBoost algorithms were $87 \%, 77 \%$ and $93 \%$ respectively. F1-score is a measure of recall and precision as suggested by Eq. 1 and it considers both false negative and false positive results. Random forest model obtained the maximum F1-score of $85 \%$. LR, KNN and XGBoost obtained $81 \%, 73 \%$ and $83 \%$ respectively. The Receiver Operator Characteristic (ROC) curve is a binary classification evaluation metric as shown in Fig. 6 . It is a probability curve that represents the true positive rate (sensitivity) against the false positive rate (1-specificity) at distinctive threshold values. These plots are created by changing the decision threshold and examining the TPR and FPR for each value. The better the model's discrimination power in the diagnostic test, the closer the area is to 1 . Random forest model obtained the optimal AUC of $91 \%$. The AUC obtained by LR, KNN and XGBoost were $89 \%, 68 \%$ and $88 \%$ respectively. Brier score is a metric for assessing the quality of a probability score that has been forecasted. This is identical to the mean squared error. However, it only applies to the prediction probability scores with the values ranging from 0 to 1 . Random forest achieved the best brier score of 0.09. The LR, KNN and XGBoost obtained a brier score of $0.15,0.25$ and 0.123 respectively.

The best random forest model used 500 decision trees (n-estimators), the maximum number of attributes while splitting the node was eight (max_features), the minimum number of samples required for internal node split (min_samples_leaf) was 1 . The maximum depth of each tree identified was 32 (max_depth). These features were identified after various iterations performed by the
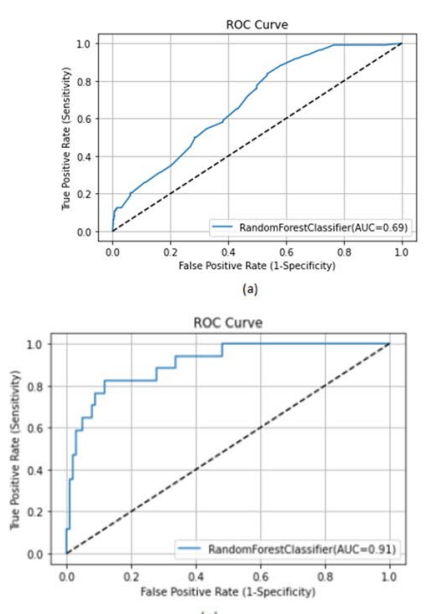

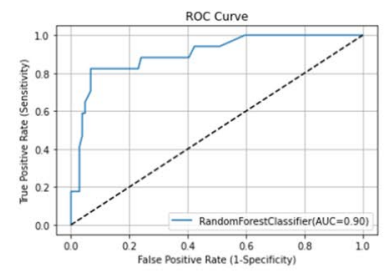

(b)

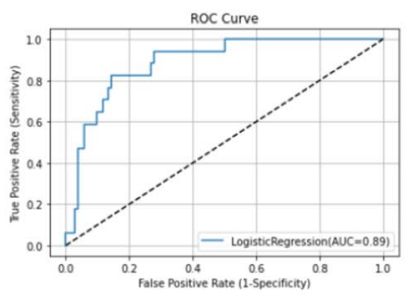

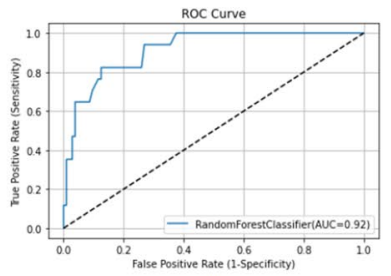

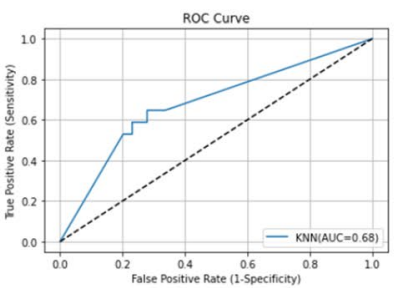

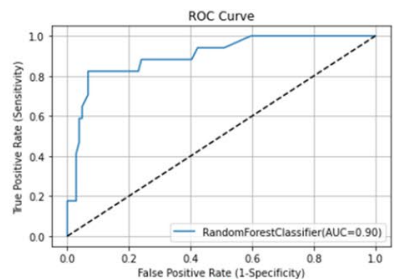

(d)

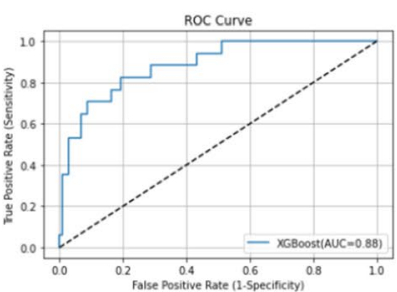

(h)

Fig. 6 AUROC curves of the various ML algorithms as follows: a Initial RF model; $\mathbf{b}$ RF model after pre-processing; $\mathbf{c}$ RF model after hyperparameter tuning; d Model after SMOTE Analysis; e Optimized RF model; f Logistic Regression; g KNN; h XGBoost 
randomizedSearchCV algorithm. The logistic regression model was also able to deliver good results. Penalty and sparsity(c) are important parameters in a logistic regression model. Generally large values of $\mathrm{c}$ give more freedom to the classifier. For our model, 100 was the optimal sparsity value along with a regularization(penalty) of 12 (Ridge regression). Ridge regression forces the weights towards zero and adds "squared magnitude" of co efficient as penalty term to the loss function. This algorithm was similar to random forest, but was slightly less able to correctly classify COVID-19 positive patients.

The KNN algorithm achieved average results. The number of nearest neighbours $(k)$ were varied and the optimal value of $\mathrm{k}$ was 2 . The distance was calculated using the Minkowski distance $(p=1)$. This distance is equivalent to Manhattan distance (If $\mathrm{p}$ was 2, Euclidean distance would have been chosen). The XGBoost classifier also achieved excellent results. The best XGBoost model used 100 decision trees(n-estimators). The maximum depth of each tree identified was eight. This boosting algorithm also uses a unique regularization parameter called "gamma". Unlike the parameters "max_depth" and "min_child_weight" that evaluates using "within tree" information, gamma uses "across trees". Hence, nodes are added only if the gain associated is large than the gamma value.

Coronavirus can be predicted using ML models as a retrospective evaluation procedure. This research identifies how ML infection models can be constructed, confirmed and utilised to quickly identify COVID-19 cases. The research also highlights the crucial significance of ML classifiers in the diagnosis and prevention of COVID-19. This helps in reducing the significant load on front line health workers and also in poor countries that suffer from lack of technology and healthcare resources.

\subsection{Feature Importance}

Glit-edge clinical judgments made using machine-learning models in healthcare settings will have an impact on patients' lives regardless of numerous legal and ethical implications. As a result, diagnostic models that are both interpretable and precise are in great demand [88], 89. Model interpretability in the medical field refers to the ability of healthcare practitioners to comprehend how the algorithm utilizes input information to make predictions and to check the classifier's outputs before taking decisions and to defend treatment decisions based on the ML models [90]. As a result, feature relevance estimations based on causality are crucial for predictive model interpretability and robustness. The Shapley Additive exPlanations (SHAP) technique [91] and random forest algorithm were used to analyse each feature's value in deciding the anticipated prediction to comprehend the suggested AI models. SHAP examines a model using Shapley values that describes how each attribute contributes to the COVID-19 prediction. Figure 7 shows a density scatter plot that reveals shapley values and combines feature relevance with effect of various features in both Sars-CoV-2 positive and negative patients. On the left, features are arranged in order of their significance. The colour on the right defines the feature value, the colour blue signifies a lower value and red signifies a higher value. Low value of leukocytes contributes the highest to the prediction model in diagnosing coronavirus positive cases, as seen in Fig. 7. Low value for eosinophils and platelets found using laboratory results in positive individuals in clinical settings are also important for our predictive model. The presence of other diseases also indicates COVID-19 negativity. In addition to this, the dots on the chart are coloured according to the normalised values of the patient's blood markers, such as the number of leukocytes. The value of a trait decreases as it gets closer to blue, and increases as it gets

Fig. 7 Feature importance using SHAP

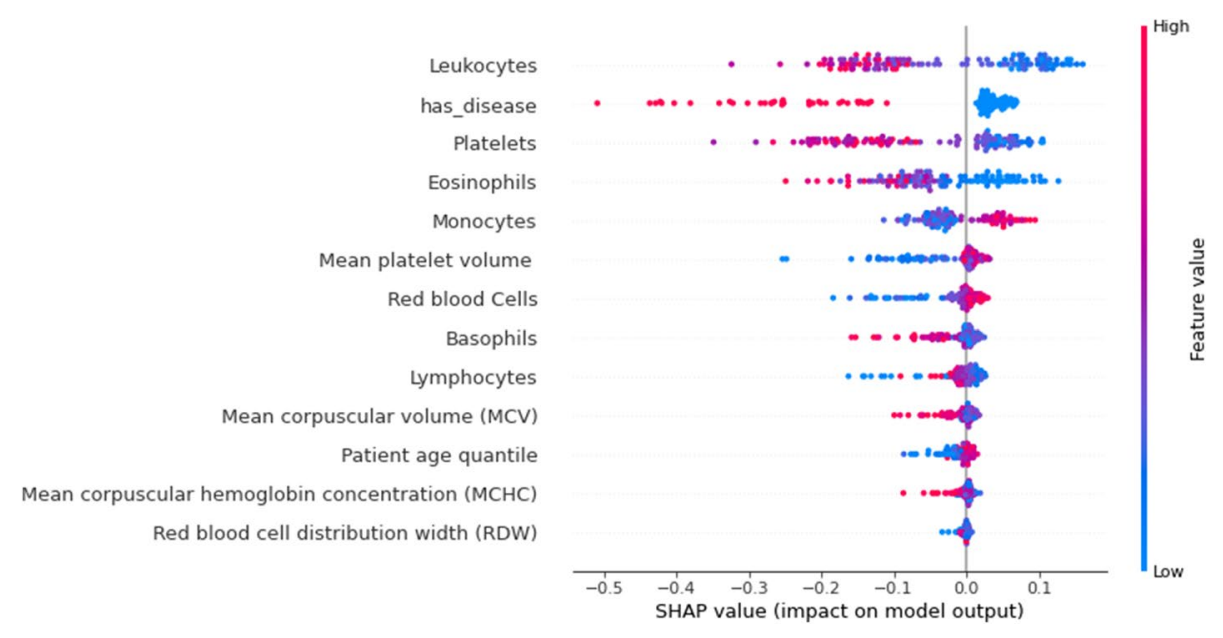




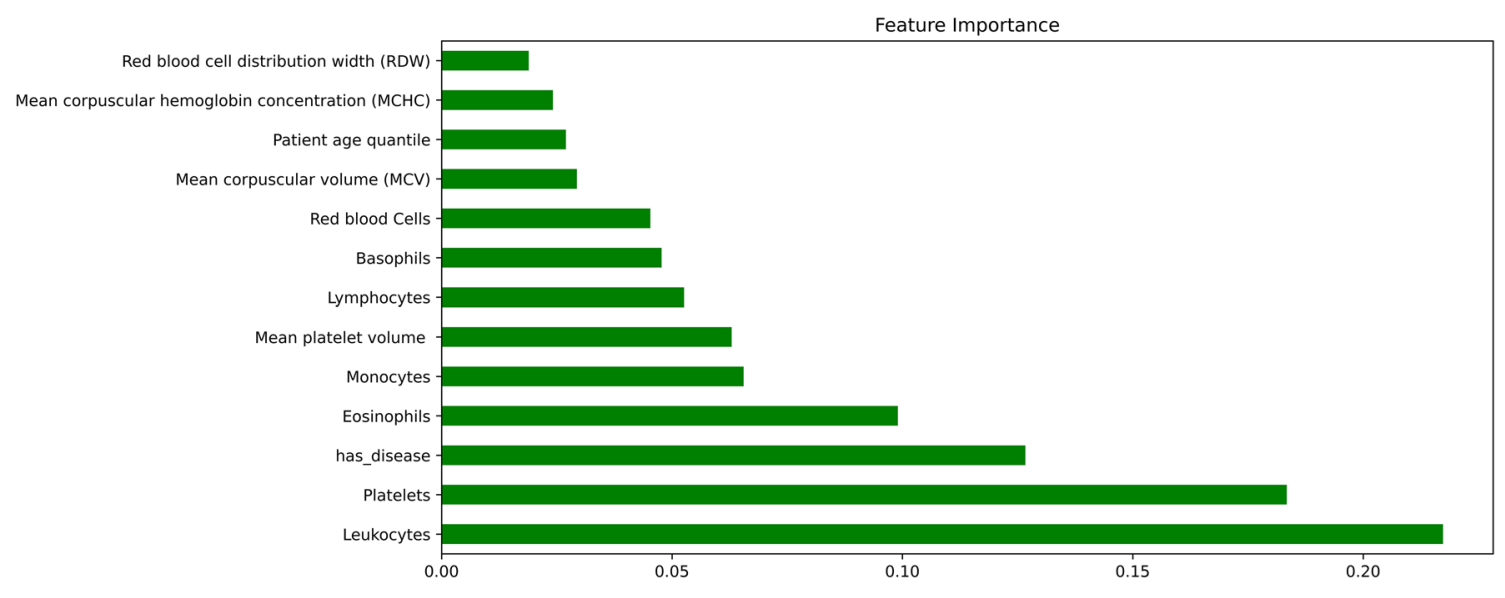

Fig. 8 Feature importance using random forest
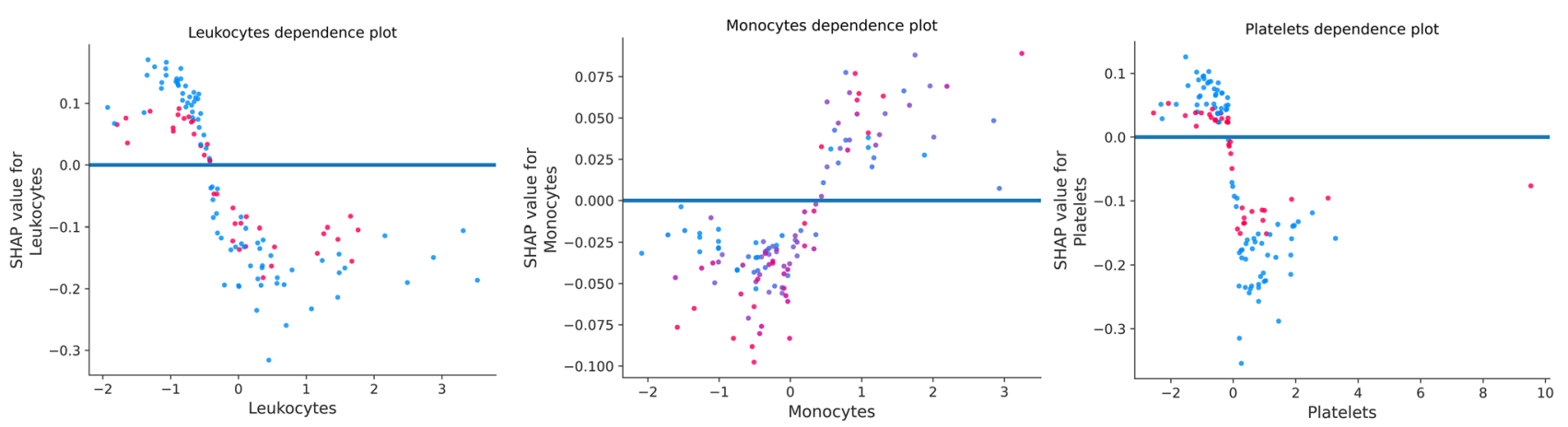

Fig. 9 Marginal effect of Leukocytes, Monocytes and Platelets on COVID-19 outcome

closer to pink. As a result, a low value of eosinophils, as well as the platelet count, as seen in blue, has a beneficial influence on the COVID-19 output.

In random forest, each predictor feature is randomly shuffled and these methods determine the importance of each attribute by monitoring the impact on model accuracy. The value of features is shown in Fig. 8 using random forest. It confirms and validates the most important features obtained from the SHAP Analysis. Figure 9 illustrates the marginal effect plot of blood markers on the target output that may be used to visualise the distribution of RT-PCR results across the sample. There is a central trend around normalised values for leukocytes and platelet levels, which are the lowest of these variables. This is in line with other researches, which suggests that platelet count can represent pathological alterations in COVID-19 cases [51]. This pattern is also observed in monocytes and appears to be linked to illness severity [52].

\subsection{Discussion on the Obtained Results}

We identified a set of routine blood test values strongly linked with SARS-CoV-2 positivity in the retrospective research of coronavirus patients along with other patients with same symptoms but verified as COVID-19 negative. These characteristics may aid doctors in identifying potential infected patients before formal diagnostic test results are available.

It has been discussed by researches that the leukocyte count tends to decrease for COVID-19 patients [78]. Our research agrees with the same and leukopenia generally occurs with lymphopenia, even when there is a normal white blood count and this condition was also associated with disease severity. The part of eosinopenia in the diagnosis of COVID-19 was discussed in many researches. Eosinophil levels were lower in patients infected with coronavirus and was also associated with patient prognosis and mortality [79]. It also has a link to coagulation disorder biomarkers as well as tissue disorder biomarkers in the kidney, liver and other tissues. Thrombocytopenia is also a common observation in COVID-19 patients. According to many studies, 
COVID-19 may trigger platelet destruction [81]. Although the cause is uncertain, it has been linked to platelet membrane components in circulating immune complexes as well as anti-platelet member GPIIa49-66 Igh antibodies. Since the current COVID-19 outbreak, multiple investigations have provided a co-relation between the infection and lymphopenia, a condition marked by abnormally low lymphocyte levels. However, it is more common in the elderly, who have a greater death risk, especially in severe cases. Lymphopenia and elevated levels of specific cytokines such as IL-6, have been linked to this devastating disease in general [76]. Monocytes are innate immune cells that participate in inflammatory reactions, phagocytosis, antigen presentation and a range of other immune function process. In our research, monocytes count increase for COVID-19 patients and agree with other conducted researches [80].

The extraordinary health catastrophe caused by the pandemic has prompted several groups of researches to create AI applications with the goal of automation in COVID-19 diagnosis and screening. Despite this, only a few AI models have been designed which is solely based on routine blood tests. Formica et al. [92] designed an AI model based on clinical and laboratory parameters with $83 \%$ sensitivity and $82 \%$ specificity. However, only a small sample was included (171). Banerjee et al. [65] used AI models on a public dataset containing 600 cases (39 positive COVID-19 patients). They found high specificity (91\%) but extremely low sensitivity (43\%) rendering it inappropriate for early detection. Avila et al. [93] designed a model that used a Bayesian approach with $76.7 \%$ sensitivity and specificity using the same dataset as [65]. Joshi et al. [94] used a CBC dataset for training a logistic regression model on a dataset that achieved higher sensitivity (93\%) and lower specificity (63\%). Finally, Yang et al., [45] in a recent study, constructed a gradient boosting model using 27 parameters (42\% were COVID-19) including both blood count and biochemical analysis. An AUC of 0.85 was reported. The summary of comparisons is given in Table 6.

To overcome the constraints of the previous models, we used machine learning to analyse the result of routine blood examinations, which are typically available for inpatients in lesser time interval and at a cheaper cost than molecular and radiographic tests. For the dataset, we used four models that are commonly deployed and adapted in medical ML. There are numerous benefits of utilizing electronic medical records, including patient information availability and security, data integration/standardization and procedural automation. Coronavirus is known to be highly contagious and quick assays to diagnose the disease are currently available. As a result, we underline that the proposed approach aimed at assisting physicians in their decision-making by offering more information. Furthermore, a significant difference of the proposed procedure is the display of model explainability, making the resources understandable to the medical personnel and thus assisting them in the final diagnosis.

\section{Key Issues and Future Directions}

This section discusses about the various challenges and the clear directions for future researches.

\subsection{Key Issues}

- Diagnosing COVID-19 from other viral infections Blood parameters such as eosinophils, platelets, leukocytes and monocytes and others vary for other viral infections too. Extensive research is required to find the parameters that can be used to distinguish coronavirus from other viral diseases. Other tests might be required to confirm the highly infectious virus.

- Single centric data The models lacked from external validation since the data belonged to a single hospital. It is very important to consider data from different geographical territories to validate the effectiveness of the models.

- Data Imbalance The data obtained is extremely imbalanced. The number of COVID-19 cases are extremely few compared to the non-COVID-19 cases. For any ML algorithm, it is very important for the data to be balanced, since balanced datasets are known to give good performance.

- Data Consistency The original values of the blood parameters are not known since the dataset was already normalized (z-normalization) by the hospital. It is extremely
Table 6 Comparison between the related studies and the proposed work

\begin{tabular}{llllll}
\hline Reference & $\begin{array}{l}\text { Accuracy of } \\
\text { best model }\end{array}$ & $\begin{array}{l}\text { Sensitivity of } \\
\text { best model }\end{array}$ & $\begin{array}{l}\text { Specificity of } \\
\text { best model }\end{array}$ & $\begin{array}{l}\text { AUC of } \\
\text { best model }\end{array}$ & ML models used \\
\hline$[92]$ & - & $83 \%$ & $82 \%$ & - & Only Statistical analysis \\
{$[65]$} & $85 \%$ & $91 \%$ & $43 \%$ & $80 \%$ & ANN, RF, glmnet \\
{$[93]$} & - & $76 \%$ & $76 \%$ & $84 \%$ & Naïve Bayes \\
{$[94]$} & - & $93 \%$ & $63 \%$ & $95 \%$ & Many models \\
{$[45]$} & - & - & - & $85 \%$ & XGBoost \\
Proposed & $91 \%$ & $94 \%$ & $71 \%$ & $91 \%$ & RF, XGBoost, LR, KNN, SVM \\
\hline
\end{tabular}


important to know the exact values for various statistical analysis.

- Lack of availability of important markers From various researches, it has been proved that various markers such as CRP, D-Dimer, LDH and ferritin are very important in diagnosing and predicting the severity of coronavirus. However, the results of those tests were not available in the dataset.

\subsection{Future Directions}

- Getting a better dataset For subsequent researches, we aim to collect a more balanced dataset. Various important blood parameters (D-dimer, LDH, CRP) should also be included. The severity of COVID-19 could also be predicted.

- Usage of Multimodal ML algorithms Ensemble algorithms are a combination of more than one base ML model that is used to improve the accuracy. Rather than creating a single model, ensemble methods consider a large number of models and combines them to produce a single final reliable classifier.

- Deep learning Unlike ML, deep learning models can perform feature engineering without external intervention. Using GPU's and TPU's will also enable a faster and efficient learning. The neural network models also work efficiently with unstructured data.

- Medical Validation After validation of the ML models by clinical experts, the models can be deployed in various health care facilities in the near future to reduce the burden on health care workers.

- Combining multiple diagnostic methods to improve accuracy These models can be used with other AI deployed models that use CT- Scans and X-ray data to improve the model performance. Integration of these models has the potential to yield optimal results.

\section{Conclusion}

COVID-19 must be identified early for patients to receive appropriate treatments and prevent the pandemic from spreading. Recent research has revealed the use of laboratory testing for preliminary patient screening, which is supported by the factuality that clinical exams are relatively less expensive, expensive and readily accessible in most treatment centres. We initially conducted an overview of current SARS-CoV-2 detection strategies utilising regular laboratory and clinical data in this article to encourage researchers to develop efficient prediction models to tackle this infectious disease. Later, multiple ML models for diagnosing COVID-19 using several clinical and laboratory markers were developed. Since four separate classifiers were utilised, structural diversity was achieved. By comparing the positive outcomes and results to the previous researches, the classifiers' effectiveness and reliability for diagnosis were also established. We used the SHAP method to assess the value of each attribute in impacting the expected result to comprehend the suggested findings better.

However, some issues must be overcome in order for ML to advance in accurate and automated COVID-19 diagnosis, especially in professional healthcare settings. High quality datasets, external validation and rigorous testing with the guidance from various doctors and healthcare personnel must be performed in the future.

Funding Open access funding provided by Manipal Academy of Higher Education, Manipal.

\section{Declarations}

Competing interests The authors declare no competing interest.

Open Access This article is licensed under a Creative Commons Attribution 4.0 International License, which permits use, sharing, adaptation, distribution and reproduction in any medium or format, as long as you give appropriate credit to the original author(s) and the source, provide a link to the Creative Commons licence, and indicate if changes were made. The images or other third party material in this article are included in the article's Creative Commons licence, unless indicated otherwise in a credit line to the material. If material is not included in the article's Creative Commons licence and your intended use is not permitted by statutory regulation or exceeds the permitted use, you will need to obtain permission directly from the copyright holder. To view a copy of this licence, visit http://creativecommons.org/licenses/by/4.0/.

\section{References}

1. WHO (2021) Coronavirus disease (covid-19). https://www.who. int/emergencies/diseases/novel-coronavirus-2019. Accessed 18 Dec 2021

2. Corman VM, Landt O, Kaiser M, Molenkamp R, Meijer A, Chu DK, Bleicker T, Brünink S, Schneider J, Schmidt ML, Mulders DG (2020) Detection of 2019 novel coronavirus (2019-nCoV) by real-time RT-PCR. Eurosurveillance 25:2000045. https://doi.org/ 10.2807/1560-7917.es.2020.25.3.2000045

3. Döhla M, Boesecke C, Schulte B, Diegmann C, Sib E, Richter E, Eschbach-Bludau M, Aldabbagh S, Marx B, Eis-Hübinger AM, Schmithausen RM (2020) Rapid point-of-care testing for SARSCoV-2 in a community screening setting shows low sensitivity. Public Health 180:170-172. https://doi.org/10.1016/j.puhe.2020. 04.009

4. Burog AI, Yacapin CP, Maglente RR, Macalalad-Josue AA, Uy EJ, Dans AL, Dans LF (2020) Should IgM/IgG rapid test kit be used in the diagnosis of COVID-19. Asia Pac Center Evid Based Healthc 4:1-12. https://doi.org/10.47895/amp.v54i0.1558

5. Browning L, Colling R, Rakha E, Rajpoot N, Rittscher J, James JA, Salto-Tellez M, Snead DR, Verrill C (2021) Digital pathology and artificial intelligence will be key to supporting clinical and academic cellular pathology through COVID-19 and future crises: 
the PathLAKE consortium perspective. J Clin Pathol 74(7):443447. https://doi.org/10.1136/jclinpath-2020-206854

6. Chamola V, Hassija V, Gupta V, Guizani M (2020) A comprehensive review of the COVID-19 pandemic and the role of IoT, drones, AI, blockchain, and $5 \mathrm{G}$ in managing its impact. Ieee access 8:90225-90265. https://doi.org/10.1109/ACCESS.2020. 2992341

7. Dash S, Chakraborty C, Giri SK, Pani SK (2021) Intelligent computing on time-series data analysis and prediction of COVID-19 pandemics. Pattern Recogn Lett 151:69-75. https://doi.org/10. 1016/j.patrec.2021.07.027

8. Rahman A, Chakraborty C, Anwar A, Karim M, Islam M, Kundu D, Rahman Z, Band SS (2021) SDN-IoT empowered intelligent framework for industry 4.0 applications during COVID19 pandemic. Clust Comput 29:1-8. https://doi.org/10.1007/ s10586-021-03367-4

9. Chakraborty C, Abougreen AN (2021) Intelligent internet of things and advanced machine learning techniques for COVID19. EAI Endors Trans Pervasive Health Technol 7:26. https://doi. org/10.4108/eai.28-1-2021.168505

10. Sajid MR, Muhammad N, Zakaria R, Shahbaz A, Bukhari SA, Kadry S, Suresh A (2021) Nonclinical features in predictive modeling of cardiovascular diseases: a machine learning approach. Interdiscip Sci 13(2):201-211. https://doi.org/10.1007/ s12539-021-00423-w

11. Orrù $\mathrm{G}$, Monaro $\mathrm{M}$, Conversano $\mathrm{C}$, Gemignani $\mathrm{A}$, Sartori $\mathrm{G}$ (2021) Machine learning in psychometrics and psychological research. Front Psychol 10:2970. https://doi.org/10.3389/fpsyg. 2019.02970

12. Rosenbusch H, Soldner F, Evans AM, Zeelenberg M (2021) Supervised machine learning methods in psychology: a practical introduction with annotated R code. Soc Pers Psychol Compass 15(2):e12579. https://doi.org/10.31234/osf.io/s72vu

13. Dhiman G, Kumar VV, Kaur A, Sharma A (2021) DON: deep learning and optimization-based framework for detection of novel coronavirus disease using X-ray images. Interdiscipl Sci 15:1-3. https://doi.org/10.1007/s12539-021-00418-7

14. Zheng F, Li L, Zhang X, Song Y, Huang Z, Chong Y, Chen Z, Zhu H, Wu J, Chen W, Lu Y (2021) Accurately discriminating COVID-19 from viral and bacterial pneumonia according to CT images via deep learning. Interdiscip Sci 13(2):273-285. https:// doi.org/10.1007/s12539-021-00420-z

15. Rasheed J, Jamil A, Hameed AA, Al-Turjman F, Rasheed A (2021) COVID-19 in the age of artificial intelligence. A comprehensive review. Interdiscip Sci. https://doi.org/10.1007/ s12539-021-00431-w

16. Abderrahim E, Xavier D, Zakaria L, Olivier L (2014) Nonlocal infinity Laplacian equation on graphs with applications in image processing and machine learning. Math Comput Simul 102:153163. https://doi.org/10.1016/j.matcom.2014.01.007

17. Hindman $M$ (2015) Building better models: prediction, replication, and machine learning in the social sciences. Ann Am Acad Polit Soc Sci 659(1):48-62

18. Grimmer J, Roberts ME, Stewart BM (2021) Machine learning for social science: an agnostic approach. Annu Rev Polit Sci 24:395419. https://doi.org/10.1146/annurev-polisci-053119-015921

19. Chen NC, Drouhard M, Kocielnik R, Suh J, Aragon CR (2018) Using machine learning to support qualitative coding in social science: shifting the focus to ambiguity. ACM Trans Interact Intell Syst 8(2):1-20. https://doi.org/10.1145/3185515

20. D'Souza S, Prema KV, Balaji S (2020) Machine learning models for drug-target interactions: current knowledge and future directions. Drug Discovery Today 25(4):748-756. https://doi.org/10. 1016/j.drudis.2020.03.003

21. Latif S, Usman M, Manzoor S, Iqbal W, Qadir J, Tyson G, Castro I, Razi A, Boulos MN, Weller A, Crowcroft J (2020) Leveraging data science to combat covid-19: a comprehensive review. IEEE Trans Artif Intell 1(1):85-103. https://doi.org/10.36227/techrxiv. 12212516

22. Pathan S, Siddalingaswamy PC, Kumar P, Manohara Pai MM, Ali T, Acharya UR (2021) Novel ensemble of optimized CNN and dynamic selection techniques for accurate Covid-19 screening using chest CT images. Comput Biol Med 137:104835. https://doi. org/10.1016/j.compbiomed.2021.104835

23. Nguyen TT, Nguyen QV, Nguyen DT, Hsu EB, Yang S, Eklund P (2020) Artificial intelligence in the battle against coronavirus (COVID-19): a survey and future research directions. arXiv 2008:07343

24. Pathan S, Siddalingaswamy PC, Ali T (2021) Automated Detection of Covid-19 from Chest X-ray scans using an optimized CNN architecture. Appl Soft Comput 104:107238. https://doi.org/10. 1016/j.asoc.2021.107238

25. Shi F, Wang J, Shi J, Wu Z, Wang Q, Tang Z, He K, Shi Y, Shen D (2020) Review of artificial intelligence techniques in imaging data acquisition, segmentation, and diagnosis for COVID-19. IEEE Rev Biomed Eng 14:4-15. https://doi.org/10.1109/rbme. 2020.2987975

26. Coppock H, Gaskell A, Tzirakis P, Baird A, Jones L, Schuller B (2021) End-to-end convolutional neural network enables COVID19 detection from breath and cough audio: a pilot study. BMJ Innov 7:356-362. https://doi.org/10.1136/bmjinnov-2021-000668

27. Tena A, Clarià F, Solsona F (2022) Automated detection of COVID-19 cough. Biomed Signal Process Control 71:103175. https://doi.org/10.1016/j.bspc.2021.103175

28. Coppock H, Jones L, Kiskin I, Schuller B (2021) COVID-19 detection from audio: seven grains of salt. Lancet Digit Health 3(9):e537-e538. https://doi.org/10.1016/s2589-7500(21)00141-2

29. Akhtar A, Akhtar S, Bakhtawar B, Kashif AA, Aziz N, Javeid MS (2021) COVID-19 detection from CBC using machine learning techniques. Int J Technol Innov Manage. 1(2):65-78. https://doi. org/10.54489/ijtim.v1i2.22

30. Ferrari D, Motta A, Strollo M, Banfi G, Locatelli M (2020) Routine blood tests as a potential diagnostic tool for COVID-19. Clin Chem Lab Med 58(7):1095-1099. https://doi.org/10.1515/ cclm-2020-0398

31. Alballa N, Al-Turaiki I (2021) Machine learning approaches in COVID-19 diagnosis, mortality, and severity risk prediction: a review. Inform Med Unlocked 3:100564. https://doi.org/10.1016/j. imu.2021.100564

32. Chadaga K, Prabhu S, Vivekananda BK, Niranjana S, Umakanth S (2021) Battling COVID-19 using machine learning: a review. Cogent Eng 8(1):1958666. https://doi.org/10.1080/23311916. 2021.1958666

33. AlJame M, Ahmad I, Imtiaz A, Mohammed A (2020) Ensemble learning model for diagnosing COVID-19 from routine blood tests. Inform Med Unlocked 21:100449. https://doi.org/10.1016/j. imu.2020.100449

34. Alves MA, Castro GZ, Oliveira BA, Ferreira LA, Ramírez JA, Silva R, Guimarães FG (2021) Explaining machine learning based diagnosis of COVID-19 from routine blood tests with decision trees and criteria graphs. Comput Biol Med 132:104335. https:// doi.org/10.1016/j.compbiomed.2021.104335

35. Muhammad LJ, Algehyne EA, Usman SS, Ahmad A, Chakraborty C, Mohammed IA (2021) Supervised machine learning models for prediction of COVID-19 infection using epidemiology dataset. SN Comput Sci 2(1):1-3. https://doi.org/10.1007/ s42979-020-00394-7

36. Brinati D, Campagner A, Ferrari D, Locatelli M, Banfi G, Cabitza F (2020) Detection of COVID-19 infection from routine blood exams with machine learning: a feasibility study. J Med Syst 44(8):1-2. https://doi.org/10.1101/2020.04.22.20075143 
37. Soares F. A novel specific artificial intelligence-based method to identify COVID-19 cases using simple blood exams. MedRxiv.

38. Schwab P, Schütte AD, Dietz B, Bauer S (2020) Clinical predictive models for COVID-19: systematic study. J Med Internet Res 22(10):e21439. https://doi.org/10.2196/preprints.21439

39. Cabitza F, Campagner A, Ferrari D, Di Resta C, Ceriotti D, Sabetta E, Colombini A, De Vecchi E, Banfi G, Locatelli M, Carobene A (2021) Development, evaluation, and validation of machine learning models for COVID-19 detection based on routine blood tests. Clin Chem Lab Med 59(2):421-431. https://doi. org/10.1515/cclm-2020-1294

40. Surkova E, Nikolayevskyy V, Drobniewski F (2020) False-positive COVID-19 results: hidden problems and costs. Lancet Respir Med 8(12):1167-1168. https://doi.org/10.1016/s2213-2600(20) 30453-7

41. Oulefki A, Agaian S, Trongtirakul T, Laouar AK (2021) Automatic COVID-19 lung infected region segmentation and measurement using CT-scans images. Pattern Recogn 114:107747. https:// doi.org/10.1016/j.patcog.2020.107747

42. Hao W, Li M (2020) Clinical diagnostic value of CT imaging in COVID-19 with multiple negative RT-PCR testing. Travel Med Infect Dis 34:101627. https://doi.org/10.1016/j.tmaid.2020. 101627

43. Shaverdian N, Shepherd AF, Rimner A, Wu AJ, Simone CB II, Gelblum DY, Gomez DR (2020) Need for caution in the diagnosis of radiation pneumonitis during the covid-19 pandemic. Adv Radiat Oncol 5(4):617-620. https://doi.org/10.1016/j.adro.2020. 04.015

44. Ismael AM, Şengür A (2021) Deep learning approaches for COVID-19 detection based on chest X-ray images. Expert Syst Appl 164:114054. https://doi.org/10.1016/j.eswa.2020.114054

45. Yang HS, Hou Y, Vasovic LV, Steel PA, Chadburn A, RacineBrzostek SE, Velu P, Cushing MM, Loda M, Kaushal R, Zhao Z (2020) Routine laboratory blood tests predict SARS-CoV-2 infection using machine learning. Clin Chem 66(11):1396-1404. https://doi.org/10.1093/clinchem/hvaa200

46. Li WT, Ma J, Shende N, Castaneda G, Chakladar J, Tsai JC, Apostol L, Honda CO, Xu J, Wong LM, Zhang T (2020) Using machine learning of clinical data to diagnose COVID-19: a systematic review and meta-analysis. BMC Med Inform Decis Mak 20(1):1-3. https://doi.org/10.1186/s12911-020-01266-z

47. Lesbon JC, Poleti MD, de Mattos Oliveira EC, Patané JS, Clemente LG, Viala VL, Ribeiro G, Giovanetti M, de Alcantara LC, de Lima LP, Nucleocapsid MAJ (2021) Gene mutations of SARSCoV-2 can affect real-time RT-PCR diagnostic and impact falsenegative results. Viruses 13(12):2474

48. Bayat V, Phelps S, Ryono R, Lee C, Parekh H, Mewton J, Sedghi F, Etminani P, Holodniy M (2020) A SARS-CoV-2 prediction model from standard laboratory tests. Clin Infect Dis 73(9):e2901-e2907. https://doi.org/10.1093/cid/ciaa1175

49. Wu J, Zhang P, Zhang L, Meng W, Li J, Tong C, Li Y, Cai J, Yang Z, Zhu J, Zhao M (2020) Rapid and accurate identification of COVID-19 infection through machine learning based on clinical available blood test results. MedRxiv.

50. Kukar M, Gunčar G, Vovko T, Podnar S, Černelč P, Brvar M, Zalaznik M, Notar M, Moškon S, Notar M (2021) COVID-19 diagnosis by routine blood tests using machine learning. Sci Rep 11(1):1-9. https://doi.org/10.1038/s41598-021-90265-9

51. Fernandes FT, de Oliveira TA, Teixeira CE, de Moraes Batista AF, Dalla Costa G, Chiavegatto Filho AD (2021) A multipurpose machine learning approach to predict COVID-19 negative prognosis in São Paulo, Brazil. Sci Rep 11(1):1-7. https://doi.org/10. 1038/s41598-021-82885-y

52. Plante TB, Blau AM, Berg AN, Weinberg AS, Jun IC, Tapson VF, Kanigan TS, Adib AB (2020) Development and external validation of a machine learning tool to rule out COVID-19 among adults in the emergency department using routine blood tests: a large, multicenter, real-world study. J Med Internet Res 22(12):e24048. https://doi.org/10.2196/preprints.24048

53. Arpaci I, Huang S, Al-Emran M, Al-Kabi MN, Peng M (2021) Predicting the COVID-19 infection with fourteen clinical features using machine learning classification algorithms. Multimedia Tools Appl 80(8):11943-11957. https://doi.org/10.1007/ s11042-020-10340-7

54. dos Santos Santana ÍV, da Silveira AC, Sobrinho Á, Silva LC, da Silva LD, Santos DF, Gurjão EC, Perkusich A (2021) Classification models for COVID-19 test prioritization in Brazil: machine learning approach. J Med Internet Res 23(4):e27293. https://doi. org/10.2196/preprints.27293

55. Goodman-Meza D, Rudas A, Chiang JN, Adamson PC, Ebinger J, Sun N, Botting P, Fulcher JA, Saab FG, Brook R, Eskin E (2020) A machine learning algorithm to increase COVID-19 inpatient diagnostic capacity. PLoS ONE 15(9):e0239474. https://doi.org/ 10.1371/journal.pone.0239474

56. Gangloff C, Rafi S, Bouzillé G, Soulat L, Cuggia M (2021) Machine learning is the key to diagnose COVID-19: a proofof-concept study. Sci Rep 11(1):1-1. https://doi.org/10.1038/ s41598-021-86735-9

57. de Freitas Barbosa VA, Gomes JC, de Santana MA, Jeniffer ED, de Souza RG, de Souza RE, dos Santos WP (2021) Heg. IA: an intelligent system to support diagnosis of Covid-19 based on blood tests. Res Biomed Eng 7:1-8. https://doi.org/10.1007/ s42600-020-00112-5

58. Rikan SB, Azar AS, Ghafari A, Mohasefi JB, Pirnejad H (2022) COVID-19 diagnosis from routine blood tests using Artificial Intelligence techniques. Biomed Signal Process Control 72:103263. https://doi.org/10.1016/j.bspc.2021.103263

59. Nan SN, Ya Y, Ling TL, Nv GH, Ying PH, Bin J (2020) A prediction model based on machine learning for diagnosing the early COVID-19 patients. medRxiv

60. Li WT, Ma J, Shende N, Castaneda G, Chakladar J, Tsai JC, Apostol L, Honda CO, Xu J, Wong LM, Zhang T (2020) Using machine learning of clinical data to diagnose covid-19. medRxiv

61. Meng Z, Wang M, Song H, Guo S, Zhou Y, Li W, Zhou Y, Li M, Song X, Zhou Y, Li Q (2020) Development and utilization of an intelligent application for aiding COVID-19 diagnosis. medRxiv

62. Xu W, Sun NN, Gao HN, Chen ZY, Yang Y, Ju B, Tang LL (2021) Risk factors analysis of COVID-19 patients with ARDS and prediction based on machine learning. Sci Rep 11(1):1-2. https://doi. org/10.1038/s41598-021-82492-x

63. Abdulkareem KH, Mohammed MA, Salim A, Arif M, Geman O, Gupta D, Khanna A (2021) Realizing an effective COVID-19 diagnosis system based on machine learning and IOT in smart hospital environment. IEEE Internet Things J 8(21):15919-15928. https://doi.org/10.1109/jiot.2021.3050775

64. Willette AA, Willette SA, Wang Q, Pappas C, Klinedinst BS, Le S, Larsen B, Pollpeter A, Li T, Brenner N, Waterboer T (2021) Using machine learning to predict COVID-19 infection and severity risk among 4,510 aged adults: a UK Biobank cohort study. medRxiv.

65. Banerjee A, Ray S, Vorselaars B, Kitson J, Mamalakis M, Weeks S, Baker M, Mackenzie LS (2020) Use of machine learning and artificial intelligence to predict SARS-CoV-2 infection from full blood counts in a population. Int Immunopharmacol 86:106705. https://doi.org/10.1016/j.intimp.2020.106705

66. Darapaneni N, Gupta M, Paduri AR, Agrawal R, Padasali S, Kumari A, Purushothaman P (2021) A Novel machine learning based screening method for high-risk Covid-19 patients based on simple blood exams. IEEE International IOT, Electronics and 
Mechatronics Conference (IEMTRONICS) (pp. 1-6). https://doi. org/10.1109/iemtronics52119.2021.9422534

67. Tschoellitsch T, Dünser M, Böck C, Schwarzbauer K, Meier J (2021) Machine learning prediction of sars-cov-2 polymerase chain reaction results with routine blood tests. Lab Med 52(2):146-149. https://doi.org/10.1093/labmed/lmaa111

68. Delafiori J, Navarro LC, Siciliano RF, De Melo GC, Busanello EN, Nicolau JC, Sales GM, De Oliveira AN, Val FF, De Oliveira DN, Eguti A (2021) Covid-19 automated diagnosis and risk assessment through metabolomics and machine learning. Anal Chem 93(4):2471-2479. https://doi.org/10.1021/acs.analchem. 0c04497.s001

69. Ning W, Lei S, Yang J, Cao Y, Jiang P, Yang Q, Zhang J, Wang X, Chen F, Geng Z, Xiong L (2020) Open resource of clinical data from patients with pneumonia for the prediction of COVID-19 outcomes via deep learning. Nat Biomed Eng 4(12):1197-1207. https://doi.org/10.1038/s41551-020-00633-5

70. Soltan AA, Kouchaki S, Zhu T, Kiyasseh D, Taylor T, Hussain ZB, Peto T, Brent AJ, Eyre DW, Clifton DA (2021) Rapid triage for COVID-19 using routine clinical data for patients attending hospital: development and prospective validation of an artificial intelligence screening test. Lancet Digit Health 3(2):78-87. https://doi.org/10.1016/s2589-7500(20)30274-0

71. Silveira EC (2020) Prediction of covid-19 from hemogram results and age using machine learning. Frontiers in Health Informatics. 9(1):39. https://doi.org/10.30699/fhi.v9i1.234

72. Singh RK, Sinha S, Ramasamy A, Kannan S, Tambi G, Basu M (2020) COVID-19 AI diagnostic tool using only 13 common blood parameters. Int J Inf Technol 6(5):220-225. https://doi.org/ 10.33144/24545414/IJIT-V6I6P1

73. Kaggle (2020), Einstein Data4u. Accessed 22 June 2021, https:// www.kaggle.com/einsteindata4u/covid19/version/4

74. Zhong Q, Peng J (2021) Mean platelet volume/platelet count ratio predicts severe pneumonia of COVID-19. J Clin Lab Anal 35(1):e23607. https://doi.org/10.1002/jcla.23607

75. Khartabil TA, Russcher H, van der Ven A, De Rijke YB (2020) A summary of the diagnostic and prognostic value of hemocytometry markers in COVID-19 patients. Crit Rev Clin Lab Sci 57(6):415-431. https://doi.org/10.1080/10408363.2020.1774736

76. Maddani SS, Gupta N, Umakanth S, Joylin S, Saravu K (2021) Neutrophil-lymphocyte ratio as a simple tool to predict requirement of admission to a critical care unit in patients with COVID19. Indian J Crit Care Med 25(5):536-539. https://doi.org/10. 5005/jp-journals-10071-23801

77. Dai W, Ke PF, Li ZZ, Zhuang QZ, Huang W, Wang Y, Xiong Y, Huang XZ (2021) Establishing classifiers with clinical laboratory indicators to distinguish COVID-19 from communityacquired pneumonia: retrospective cohort study. J Med Internet Res 23(2):e23390. https://doi.org/10.2196/23390

78. Kahn R, Schmidt T, Golestani K, Mossberg A, Gullstrand B, Bengtsson AA, Kahn F (2021) Mismatch between circulating cytokines and spontaneous cytokine production by leukocytes in hyperinflammatory COVID-19. J Leukoc Biol 109(1):115-120. https://doi.org/10.1002/jlb.5covbcr0720-310rr

79. Tabachnikova A, Chen ST (2020) Roles for eosinophils and basophils in COVID-19? Nat Rev Immunol 20(8):461-474. https://doi. org/10.1038/s41577-020-0379-1
80. Gómez-Rial J, Rivero-Calle I, Salas A, Martinón-Torres F (2020) Role of monocytes/macrophages in Covid-19 pathogenesis: implications for therapy. Infect Drug Resist 13:2485-2489. https://doi. org/10.2147/IDR.S258639

81. Thachil J (2020) What do monitoring platelet counts in COVID-19 teach us? J Thromb Haemost 18(8):2071-2072. https://doi.org/10. 1111/j.1538-7836.2011.04279.x

82. Oshiro TM, Perez PS, Baranauskas JA (2012) How many trees in a random forest?. In: International workshop on machine learning and data mining in pattern recognition (pp. 154-168). Springer, Berlin, Heidelberg. https://doi.org/10.1007/978-3-642-31537-4_ 13

83. Peterson LE (2009) K-nearest neighbor. Scholarpedia. https://doi. org/10.4249/scholarpedia.1883

84. Chen T, He T, Benesty M, Khotilovich V, Tang Y, Cho H (2015) Xgboost: extreme gradient boosting. $\mathrm{R}$ package version $0.4-2$. https://doi.org/10.1145/2939672.2939785

85. Menard S (2002) Applied logistic regression analysis. Sage, New York. https://doi.org/10.4135/9781412983433

86. De Cock M, Dowsley R, Nascimento AC, Railsback D, Shen J, Todoki A (2021) High performance logistic regression for privacy-preserving genome analysis. BMC Med Genomics 14(1):18. https://doi.org/10.1186/s12920-020-00869-9

87. Fernández A, Garcia S, Herrera F, Chawla NV (2018) SMOTE for learning from imbalanced data: progress and challenges, marking the 15-year anniversary. J Artif Intell Res 61:863-905. https://doi. org/10.1613/jair.1.11192

88. Pedregosa F, Varoquaux G, Gramfort A, Michel V, Thirion B, Grisel O, Blondel M, Prettenhofer P, Weiss R, Dubourg V, Vanderplas J (2011) Scikit-learn: machine learning in Python. J Mach Learn Res 12:2825-2830. https://doi.org/10.5555/1953048.20781 95

89. Molnar C (2019) Interpretable machine learning. https://chris tophm.github.io/interpretable-ml-book/

90. Holzinger A, Langs G, Denk H, Zatloukal K, Müller H (2019) Causability and explainability of artificial intelligence in medicine. Wiley Interdiscip Rev 9:e1312. https://doi.org/10.1002/ widm. 1312

91. Parsa AB, Movahedi A, Taghipour H, Derrible S, Mohammadian AK (2020) Toward safer highways, application of XGBoost and SHAP for real-time accident detection and feature analysis. Accid Anal Prev 136:105405. https://doi.org/10.1016/j.aap.2019.105405

92. Formica V, Minieri M, Bernardini S, Ciotti M, D’Agostini C, Roselli M, Andreoni M, Morelli C, Parisi G, Federici M, Paganelli C (2020) Complete blood count might help to identify subjects with high probability of testing positive to SARS-CoV-2. Clin Med 20(4):e114. https://doi.org/10.7861/clinmed.2020-0373

93. Avila E, Kahmann A, Alho C, Dorn M (2020) Hemogram data as a tool for decision-making in COVID-19 management: applications to resource scarcity scenarios. PeerJ 8:e9482. https://doi.org/ 10.7717/peerj.9482

94. Joshi RP, Pejaver V, Hammarlund NE, Sung H, Lee SK, Furmanchuk AO, Lee HY, Scott G, Gombar S, Shah N, Shen S (2020) A predictive tool for identification of SARS-CoV-2 PCR-negative emergency department patients using routine test results. J Clin Virol 129:104502. https://doi.org/10.1016/j.jcv.2020.104502 


\section{Authors and Affiliations}

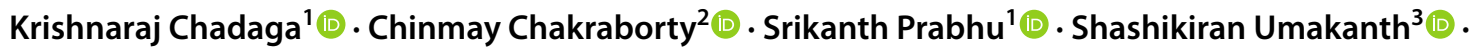
Vivekananda Bhat ${ }^{1}\left[{ }^{\mathbb{D}} \cdot\right.$ Niranjana Sampathila $^{4}(\mathbb{D}$

1 Department of Computer Science and Engineering, Manipal Institute of Technology, Manipal Academy of Higher Education, Manipal, India

2 Department of Electronics and Communication, Birla Institute of Technology, Mesra, India
3 Department of Medicine, Dr. TMA Hospital, Manipal Academy of Higher Education, Manipal, India

4 Department of Biomedical Engineering, Manipal Institute of Technology, Manipal Academy of Higher Education, Manipal, India 